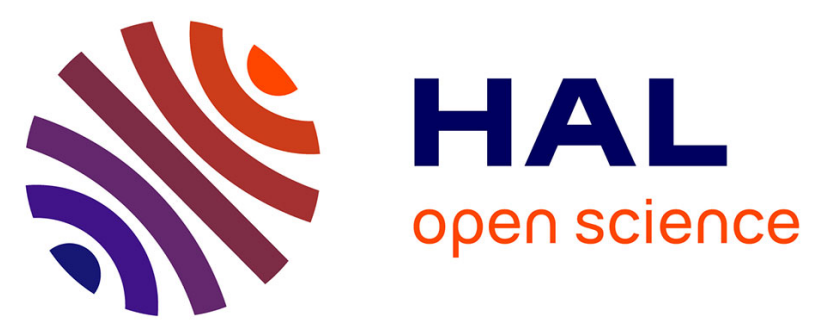

\title{
Theory and applications of time reversal and interferometric imaging
}

Liliana Borcea, George Papanicolaou, Chrysoula Tsogka

\section{To cite this version:}

Liliana Borcea, George Papanicolaou, Chrysoula Tsogka. Theory and applications of time reversal and interferometric imaging. Inverse Problems, 2003, 19 (6), pp.139-164. 10.1088/0266-5611/19/6/058 . hal-00106406

\section{HAL Id: hal-00106406 https://hal.science/hal-00106406}

Submitted on 1 Feb 2022

HAL is a multi-disciplinary open access archive for the deposit and dissemination of scientific research documents, whether they are published or not. The documents may come from teaching and research institutions in France or abroad, or from public or private research centers.
L'archive ouverte pluridisciplinaire HAL, est destinée au dépôt et à la diffusion de documents scientifiques de niveau recherche, publiés ou non, émanant des établissements d'enseignement et de recherche français ou étrangers, des laboratoires publics ou privés.

\section{다)(1) $(5$}

Distributed under a Creative Commons Attribution - NonCommercial| 4.0 International 


\title{
Theory and applications of time reversal and interferometric imaging
}

\author{
Liliana Borcea* $\quad$ George Papanicolaou ${ }^{\dagger} \quad$ Chrysoula Tsogka ${ }^{\ddagger}$
}

September 30, 2003

\begin{abstract}
In time reversal, an array of transducers receives the signal emitted by a localized source, time reverses it and re-emits it into the medium. The emitted waves back-propagate to the source and tend to focus near it. In a homogeneous medium, the cross-range resolution of the refocused field at the source location is $\lambda_{0} L / a$, where $\lambda_{0}$ is the carrier wavelength, $L$ is the range and $a$ is the array aperture. The refocusing spot size in a homogeneous medium is independent of the bandwidth of the pulse, but broadband can help in reducing spurious Fresnel zones. In a noisy (random) medium, the cross-range resolution is improved beyond the homogeneous diffraction limit because the array can capture waves that move away from it at the source, but get scattered onto it by the inhomogeneities. We refer to this phenomenon as super-resolution of the time reversal process in random media. Super-resolution implies in particular that, because of multipathing, the array appears to have an effective aperture $a_{e}$ that is greater than $a$. Since $a_{e}$ depends on the scattering medium, it is not known. In this paper we present a brief review of time reversal theory in a remote sensing regime and a robust procedure for estimating $a_{e}$ from the signals received at the array. Knowing $a_{e}$ permits assessing quantitatively super-resolution in time reversal for applications in spatially localized communications with reduced interference. We also review interferometric imaging and its relation to time reversal and to matched field imaging. We show that $a_{e}$ quantifies in an explicit way the loss of resolution in interferometric array imaging.
\end{abstract}

PACS numbers: 43.60.Gk, 43.60.Cg, 43.60.Rw, $43.60 \mathrm{Tj}$

\section{Introduction}

There have been recently some theoretical and experimental developments in time reversal, where signals emitted by a source are recorded by an array of transducers, time reversed and sent back into the same medium. Because of the time reversibility of the wave equation we have (diffraction limited) refocusing of the time reversed signal at the source when there is no attenuation. In a homogeneous medium we get a refocusing spot of approximate size $\lambda_{0} L / a$ [14]. However, in random

\footnotetext{
${ }^{*}$ Computational and Applied Mathematics, MS 134, Rice University, 6100 Main Street, Houston, TX 770051892.(borcea@caam.rice.edu)

${ }^{\dagger}$ Department of Mathematics, Stanford University, Stanford, CA 94305. (papanico@math.stanford.edu)

${ }^{\ddagger}$ CNRS/LMA, 31 Chemin Joseph Aiguier, 13402 Marseille cedex 20, FRANCE, (tsogka@lma.cnrs-mrs.fr)
} 
media experimental $[21,27,42,35]$ and theoretical $[8,38,2,23]$ studies show that in broadband regimes the refocusing is much tighter and the Fresnel zones are reduced. This is the phenomenon of super-resolution and it is due to the random inhomogeneities, which distribute the waves over a larger part of the medium than they would in the homogeneous case and therefore carry more information about the source location. The array appears to have an effective aperture $a_{e}>a$ and this leads to super-resolution and the elimination of Fresnel zones because of random phase cancellations. A quantitative assessment of super-resolution can be made by looking at the average time reversed back-propagated field, calculated explicitly in [23]. However, it is remarkable that in appropriate regimes, like broadband, this phenomenon does not happen just in the mean but for almost all realizations of the random medium. This is the self-averaging phenomenon which has been explored numerically and analytically in $[8,38,2]$, and for layered random media in $[18,28]$ and the references therein. Self-averaging is important because it differentiates between an observable refocusing with super-resolution and random speckle patterns distributed around the original source.

Since reflection-based array imaging methods involve some form of time reversal or backpropagation into the real (or a fictitious) medium, it is natural to ask what implications superresolution and statistical stability in time reversal have for imaging in clutter. In $[12,6]$ we developed an approach to statistically stable imaging of small targets buried in a randomly inhomogeneous, isotropic, infinite medium. In [10], we reported our first resolution study, for imaging an active point source with a passive array of transducers, in random media. However, the details of the calculations are not given in [10] and we present them here. We also introduce a resolution study of interferometric imaging of extended, quiescent objects, in clutter, from the scattered echoes received at the array. Using theoretical results of time reversal in random media [8, 38, 2, 23], we develop a model for interferometric, matched field imaging, which accounts in a simple and explicit manner for the effect of the clutter on the image. This effect is quantified by a single parameter, the effective aperture $a_{e}$, which is unknown. We show here that the matched field functional can be used not only to locate unknown sources and reflectors but also to provide an estimate for $a_{e}$, and we demonstrate the feasibility of our approach with numerical simulations. In particular, we show that the estimated $a_{e}$ predicts very accurately the spot size in time reversal in random media. Since the effective aperture $a_{e}$ depends on the scattering medium its estimation gives a measure of the randomness of the medium and plays an important role in several applications. To illustrate this, we briefly present in section 6 a reflectivity imaging method for extended scatterers in random media. We show that in this case the blurring effect of the random medium on the image can be also quantified with $a_{e}$. Another application in which $a_{e}$ appears as a key parameter is communications in cluttered media as we discuss in section 7 .

In section 2 we introduce time reversal and imaging by arrays. In section 3 we discuss time reversal in both deterministic and random media. In section 4, we discuss imaging active point sources in random media. In section 5 we use the matched field functional of section 4 to estimate the effective aperture $a_{e}$, which depends on the random medium and on the range. We demonstrate the feasibility and robustness of the estimation process with numerical simulations in section 5 . The important role that $a_{e}$ plays in imaging applications is illustrated with two examples: imaging of the reflectivity of extended targets, presented in section 6 , and secure communications, discussed in section 7 . Finally, in section 8 we give a brief summary and conclusions. 


\section{$2 \quad$ Imaging and time reversal}

For simplicity we assume that there is a single scatterer to be imaged in the noisy medium. We distinguish between two types of scatterers or targets: (1) active ones, which emit a signal $f(t)$ that propagates through the medium and is received at the array, and (2) passive ones, which are quiet and can be detected and imaged from scattered signals received at the array, that are excited by primary signals emitted by the array. The scatterers can be small, point-like, or extended but of finite support. Let us begin with the case of a small, active source, located at

$$
\mathbf{y}=(0,0, L),
$$

with respect to the center of the array, where $L$ is the range and the cross-range is zero. The array has point transducers located at $\mathbf{x}_{p}=(p h / 2,0,0)$, for $p=-N, \ldots, N$. The separation $h / 2$ between the array elements is chosen so that in a remote sensing regime the transducers behave like an array of aperture $a=N h \ll L$ and not like separate entities, while interference is kept at a minimum. Often, $h=\lambda_{0}$, the wavelength of the carrier frequency of the pulse.

Suppose that we have an active source which emits a pulse

$$
f(t)=-\frac{d}{d t}\left(\frac{1}{\sqrt{2 \pi \sigma_{t}^{2}}} e^{-i \omega_{0} t} e^{-\frac{t^{2}}{2 \sigma_{t}^{2}}}\right)=\frac{i \omega_{0}+\frac{t}{\sigma_{t}^{2}}}{\sqrt{2 \pi \sigma_{t}^{2}}} e^{-i \omega_{0} t} e^{-\frac{t^{2}}{2 \sigma_{t}^{2}}},
$$

where $\nu_{0}=\omega_{0} / 2 \pi$ is the carrier frequency and $B=1 /\left(\sigma_{t} \nu_{0}\right)$ is the (relative) bandwidth of

$$
\widehat{f}(\omega)=\int_{-\infty}^{\infty} f(t) e^{i \omega t} d t=i \omega e^{-\frac{\sigma_{t}^{2}\left(\omega-\omega_{0}\right)^{2}}{2}} .
$$

Clearly, there are many other choices for $f(t)$. For example, chirps such as $f(t)=e^{i \omega_{0} t+i \alpha t^{2}}$ are commonly used in synthetic aperture radar imaging $[30,13,37,19,16]$. We use the pulse $(2.1)$ here for simplicity in the calculations. The signal received at $\mathbf{x}_{p}$ (see Figure 1 ) is

$$
s_{p}(t)=f(t) \star_{t} G\left(\mathbf{x}_{p}, \mathbf{y}, t\right)=\frac{1}{2 \pi} \int_{-\infty}^{\infty} \widehat{f}(\omega) \widehat{G}\left(\mathbf{x}_{p}, \mathbf{y}, \omega\right) e^{-i \omega t} d \omega
$$

where $\widehat{G}$ is the two point Green's function at radian frequency $\omega$, and where $\star_{t}$ denotes convolution in time. Throughout the paper, we use the reciprocity of the Green's function which allows us to interchange its spatial arguments.

We choose the scalar wave equation as our mathematical model for wave propagation in the medium and we let $c(\mathbf{x})$ be the propagation speed at a point $\mathbf{x} \in \mathbb{R}^{3}$. This scalar model is appropriate for sonar and ultrasound regimes but not for electromagnetic waves or for seismic imaging, although it is often used there too. At frequency $\nu=\omega / 2 \pi$, the two point, outgoing Green's function satisfies the reduced wave equation

$$
\Delta \widehat{G}(\mathbf{x}, \mathbf{y}, \omega)+k^{2} n^{2}(\mathbf{x}) \widehat{G}(\mathbf{x}, \mathbf{y}, \omega)=-\delta(\mathbf{x}-\mathbf{y}),
$$

where $k=\omega / c_{0}$ is the wavenumber, $c_{0}$ is a reference speed of propagation and

$$
n(\mathbf{x})=\frac{c_{0}}{c(\mathbf{x})}
$$

is the index of refraction of the medium. 


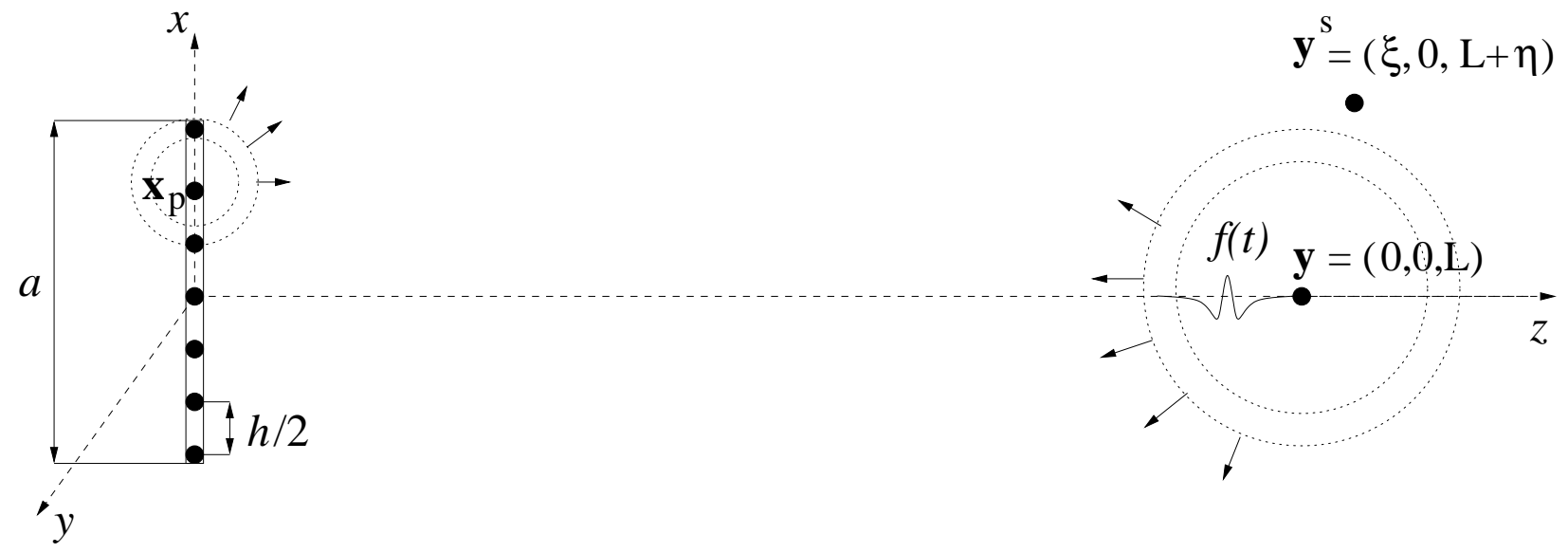

Figure 1: The setup for array time reversal and imaging

We neglect the presence of boundaries and interfaces in the medium and focus attention just on the scattering by the random inhomogeneities. For simplicity we also neglect large scale background variations, although they can be accounted for easily in numerical calculations, and we let the fluctuations of the index of refraction be

$$
n^{2}(\mathbf{x})-1=\sigma \mu\left(\frac{\mathbf{x}}{l}\right)
$$

where $l$ is the correlation length (the scale at which the medium fluctuates), the standard deviation $\sigma \ll 1$ (weak fluctuations) and $\mu$ is a stationary, isotropic random field with mean zero and normalized covariance

$$
R(\mathbf{x})=R(|\mathbf{x}|)=E\left\{\mu\left(\mathbf{x}^{\prime}+\mathbf{x}\right) \mu\left(\mathbf{x}^{\prime}\right)\right\} .
$$

We also assume that the covariance (2.7) decays sufficiently fast at infinity. We review some of the basic theory of waves in random media with such fluctuations in the appendix. Note that the scattering medium that we consider here is different than the one in the experiments in [21, 27], where the fluctuations are very strong. We take weak fluctuations but because the waves travel a long distance in the scattering medium, the multipathing effect is observable, as illustrated by the super-resolution that we get in the numerical simulations of time reversal. We show in this paper that, in such random media, the effective aperture $a_{e}$ is the only parameter needed to quantify super-resolution.

In imaging, we seek the unknown location $\mathbf{y}$ of the source that is buried in the unknown random medium by reversing in time the signals $s_{p}(t), p=-N, \ldots, N$, and back-propagating them numerically into a fictitious medium, which is here homogeneous with constant sound speed $c_{0}$. This process, which is also referred to as migration [17, 7], or back-projection [37] in geophysics and in X-ray crystallography, respectively, is a form of time reversal $[8,25,26,27,42,35,18]$. In time reversal, the signals $s_{p}(t), p=-N, \ldots, N$, received at the array are time reversed and re-emitted into the actual medium. They back-propagate to the source and focus near it.

Thus, in both imaging and time reversal, we consider the back-propagated field at a "search" point $\mathbf{y}^{s}$ as shown in Figure 1. We take $\mathbf{y}^{s}$ in the plane determined by $\mathbf{y}$ and the array, at range $L+\eta$ and cross-range $\xi$,

$$
\mathbf{y}^{s}=(\xi, 0, L+\eta)
$$


The space-time point-spread function for time reversal is defined by

$$
\Gamma^{T R}\left(\mathbf{y}^{s}, t\right)=\frac{1}{2 \pi} \int_{-\infty}^{\infty} e^{-i \omega t} \widehat{\Gamma}^{T R}\left(\mathbf{y}^{s}, \omega\right) d \omega
$$

where

$$
\widehat{\Gamma}^{T R}\left(\mathbf{y}^{s}, \omega\right)=\sum_{p=-N}^{N} \overline{\widehat{s}_{p}(\omega)} \widehat{G}\left(\mathbf{x}_{p}, \mathbf{y}^{s}, \omega\right)=\overline{\widehat{f}(\omega)} \sum_{p=-N}^{N} \overline{\widehat{G}\left(\mathbf{x}_{p}, \mathbf{y}, \omega\right)} \widehat{G}\left(\mathbf{x}_{p}, \mathbf{y}^{s}, \omega\right),
$$

and where the bar in (2.9) stands for complex conjugation. This function peaks near $t \approx 0$ and $\mathbf{y}^{s} \approx y$. The point-spread function for imaging is given by

$$
\Gamma^{I M}\left(\mathbf{y}^{s}, t=0\right)=\frac{1}{2 \pi} \int_{-\infty}^{\infty} \widehat{\Gamma}^{I M}\left(\mathbf{y}^{s}, \omega\right) d \omega
$$

where

$$
\widehat{\Gamma}^{I M}\left(\mathbf{y}^{s}, \omega\right)=\sum_{p=-N}^{N} \overline{\widehat{s}_{p}(\omega)} \widehat{G}_{0}\left(\mathbf{x}_{p}, \mathbf{y}^{s}, \omega\right)=\overline{\widehat{f}(\omega)} \sum_{p=-N}^{N} \overline{\widehat{G}\left(\mathbf{x}_{p}, \mathbf{y}, \omega\right)} \widehat{G}_{0}\left(\mathbf{x}_{p}, \mathbf{y}^{s}, \omega\right)
$$

and where $\widehat{G}_{0}\left(\mathbf{x}_{p}, \mathbf{y}^{s}, \omega\right)$ is the Green's function in a homogeneous medium

$$
\widehat{G}_{0}(\mathbf{x}, \mathbf{y}, \omega)=\frac{e^{i k|\mathbf{x}-\mathbf{y}|}}{4 \pi|\mathbf{x}-\mathbf{y}|}=\frac{e^{i \omega t(\mathbf{x}, \mathbf{y})}}{4 \pi|\mathbf{x}-\mathbf{y}|} .
$$

Note that, since $\mathbf{y}$ is constant, we suppress it from the list of arguments in $\Gamma^{T R}$ and $\Gamma^{I M}$.

In a homogeneous medium the travel time from $\mathbf{x}$ to $\mathbf{y}$ is $t(\mathbf{x}, \mathbf{y})=|\mathbf{x}-\mathbf{y}| / c_{0}$. We note that $\Gamma^{I M}$ differs from $\Gamma^{T R}$ only insofar as the back propagation is done in a homogeneous or reference medium in imaging. Up to a scaling factor that depends on range, the imaging functional (2.10) is equivalent to the Kirchhoff migration functional

$$
\Gamma^{K M}\left(\mathbf{y}^{s}\right)=\sum_{p=-N}^{N} s_{p}\left(t_{p}\left(\mathbf{y}^{s}\right)\right)
$$

where $t_{p}\left(\mathbf{y}^{s}\right)=\left|\mathbf{x}_{p}-\mathbf{y}\right| / c_{0}$ is the travel time from $\mathbf{x}_{p}$ to $\mathbf{y}^{s}$.

In this paper, we analyze the point-spread functions for time reversal and imaging, both in the frequency and in the time domain, for randomly inhomogeneous media in a weakly fluctuating, remote sensing regime. This functional, like (2.10), peaks when the search point $\mathbf{y}^{s}$ is near the unknown source location $\mathbf{y}$.

\section{Time reversal}

When dissipative effects of the medium can be neglected, the time reversal point spread function focuses spatially and temporary near the source position $\mathbf{y}$. Because the array aperture $a$ is limited, the refocusing is diffraction limited. In homogeneous media, the focal spot size is of order $\lambda_{0} L / a$ [14] and Fresnel zones may be observed, especially in a narrowband regime. It is surprising at first, that in richly scattering media we observe much better focusing [21, 25, 26, 27, 35, 42]. But there is a simple physical explanation for this. Multiple scattering (multipathing) in the medium causes rays that are directed initially away from the array, to be scattered back onto it, and thus 
to be captured, time reversed and reemited in the medium. The scattering medium makes the array appear to be larger, that is, to have an effective aperture $a_{e}>a$ and the result is superresolution with a focal spot size of order $\lambda_{0} L / a_{e}$. The Fresnel zones also disappear, because of random phase cancellations. Of course, scattering reduces signal power at the array, because rays are scattered off of it, so amplification is often needed. The other surprising effect in time reversal is that under certain conditions, for example in broad band regimes (see [8]), the refocused field is self-averaging or statistically stable. This property is what makes super-resolution in time reversal observable when the environment is richly scattering.

In what follows, we analyze the time reversal point spread function $\Gamma^{T R}$ in homogeneous and random media. The effect of the clutter on $\Gamma^{T R}$ is quantified explicitly by the effective aperture. In the frequency domain, $\widehat{\Gamma}^{T R}\left(\mathbf{y}^{s}, \omega\right)$ depends on the narrow-band effective aperture $a_{e}$ introduced in [8]. However, in broad band regimes, the focusing of $\Gamma^{T R}\left(\mathbf{y}^{s}, t\right)$ is determined by the broad-band effective aperture $A_{e}$, which depends both on $a_{e}$ and on the bandwidth of the probing pulse $f(t)$.

\subsection{Time reversal in homogeneous media}

The time reversal point-spread function in homogeneous media is

$$
\Gamma_{0}^{T R}\left(\mathbf{y}^{s}, t\right)=\frac{1}{2 \pi} \int_{-\infty}^{\infty} \widehat{\Gamma}_{0}^{T R}\left(\mathbf{y}^{s}, \omega\right) e^{-i \omega t} d \omega
$$

where

$$
\begin{aligned}
\widehat{\Gamma}_{0}^{T R}\left(\mathbf{y}^{s}, \omega\right) & =\overline{\widehat{f}(\omega)} \sum_{p=-N}^{N} \overline{\widehat{G}_{0}\left(\mathbf{x}_{p}, \mathbf{y}, \omega\right)} \widehat{G}_{0}\left(\mathbf{x}_{p}, \mathbf{y}^{s}, \omega\right) \\
& =\overline{\widehat{f}(\omega)} \sum_{p=-N}^{N} \frac{e^{i k\left(\left|\mathbf{x}_{p}-\mathbf{y}^{s}\right|-\left|\mathbf{x}_{p}-\mathbf{y}\right|\right)}}{(4 \pi)^{2}\left|\mathbf{x}_{p}-\mathbf{y}\right|\left|\mathbf{x}_{p}-\mathbf{y}^{s}\right|}
\end{aligned}
$$

In a remote sensing regime $(a \ll L)$, we can use the parabolic approximation of the phase

$$
\left|\mathbf{x}_{p}-\mathbf{y}\right|=\left(L^{2}+x_{p}^{2}\right)^{\frac{1}{2}} \approx L+\frac{x_{p}^{2}}{2 L} .
$$

Similarly, for $\mathbf{y}^{s}$ close to the target,

$$
\left|\mathbf{x}_{p}-\mathbf{y}^{s}\right|=\left[(L+\eta)^{2}+\left(\xi-x_{p}\right)^{2}\right]^{\frac{1}{2}} \approx L+\eta+\frac{\left(\xi-x_{p}\right)^{2}}{2(L+\eta)} .
$$

Then using (3.3) and (3.4) for approximating the phase in (3.2), we have $\left(\left|\mathbf{x}_{p}-\mathbf{y}^{s}\right|,\left|\mathbf{x}_{p}-\mathbf{y}\right|\right.$ are approximated by $L$ in the amplitude),

$$
\widehat{\Gamma}_{0}^{T R}(\xi, 0, \omega) \approx \frac{\bar{f}(\omega)}{(4 \pi L)^{2}} e^{i k\left(\eta+\frac{\xi^{2}}{2(L+\eta)}\right)} \sum_{p=-N}^{N} e^{-i k\left(\frac{\eta x_{p}^{2}}{2 L(L+\eta)}+\frac{x_{p} \xi}{L+\eta}\right)},
$$

and since $x_{p}=p h / 2$ and $h$ is small (with respect to $a$ and $L$ ) we approximate the sum in (3.5) by an integral

$$
\widehat{\Gamma}_{0}^{T R}(\xi, \eta, \omega) \approx \frac{\overline{\hat{f}(\omega)}}{8 \pi^{2} L^{2} h} e^{i k\left(\eta+\frac{\xi^{2}}{2(L+\eta)}\right)} \int_{-a / 2}^{a / 2} e^{-i k\left(\frac{\eta x^{2}}{2 L(L+\eta)}+\frac{x \xi}{L+\eta}\right)} d x .
$$


Finally, using the Fourier coefficients of the pulse (2.2) we obtain the time reversed, back-propagated field at the search point,

$$
\Gamma_{0}^{T R}(\xi, \eta, t) \approx-\frac{1}{2 \pi} \int_{-\infty}^{\infty} d \omega \frac{i \omega e^{-\frac{\sigma_{t}^{2}\left(\omega-\omega_{0}\right)^{2}}{2}}}{8 \pi^{2} L^{2} h} e^{-i \omega\left[t-\frac{1}{c_{0}}\left(\eta+\frac{\xi^{2}}{2(L+\eta)}\right)\right]} \int_{-a / 2}^{a / 2} d x e^{-i \frac{\omega}{c_{0}}\left(\frac{\eta x^{2}}{2 L(L+\eta)}+\frac{x \xi}{L+\eta}\right)}
$$

To estimate the spatial focusing resolution in cross-range, let us evaluate $\Gamma_{0}^{T R}$ at the exact range $\eta=0$ and at the arrival time $t=\xi^{2} /\left(2 c_{0} L\right)$,

$$
\Gamma_{0}^{T R}\left(\xi, \eta=0, t=\frac{\xi^{2}}{2 c_{0} L}\right) \approx \frac{c_{0}}{4 \pi^{2} L h \xi} \frac{-i}{\sqrt{2 \pi \sigma_{t}^{2}}} \sin \left(\frac{\omega_{0} \xi a}{2 c_{0} L}\right) e^{-\frac{a^{2} \xi^{2}}{8 c_{0}^{2} L^{2} \sigma_{t}^{2}}}
$$

For the pulse (2.1), the cross-range focusing resolution is determined by the product of $\operatorname{sinc}\left(\frac{\pi \xi a}{\lambda_{0} L}\right)=$ $\sin \left(\frac{\pi \xi a}{\lambda_{0} L}\right) /\left(\frac{\pi \xi a}{\lambda_{0} L}\right)$ and a Gaussian $e^{-\frac{\xi^{2}}{2 s_{0}^{2}}}$ with standard deviation

$$
s_{0}=\frac{2 c_{0} \sigma_{t} L}{a}=2 \nu_{0} \sigma_{t} \frac{\lambda_{0} L}{a}=\frac{2}{B} \frac{\lambda_{0} L}{a},
$$

where $\omega_{0}=2 \pi \nu_{0}$ and $B=1 /\left(\nu_{0} \sigma_{t}\right)$ is the relative bandwidth, $0<B<1$. In the case of a narrowband pulse, $s_{0}$ is large and the deterministic resolution limit $\frac{\lambda_{0} L}{a}$ comes from the sinc function. The Fresnel zones are also visible. For a broad-band pulse, $s_{0}$ is comparable to $\frac{\lambda_{0} L}{a}$, which is the spot size determined by the Gaussian factor, and the Fresnel zones are now eliminated. In either case, the larger the aperture $a$ is the better the focusing.

\subsection{Time reversal in random media}

The time reversed, back-propagated field in the random medium is

$$
\Gamma^{T R}\left(\mathbf{y}^{s}, t\right)=\frac{1}{2 \pi} \int \widehat{\Gamma}^{T R}\left(\mathbf{y}^{s}, \omega\right) e^{-i \omega t} d \omega
$$

for

$$
\widehat{\Gamma}^{T R}\left(\mathbf{y}^{s}, \omega\right)=\overline{\widehat{f}(\omega)} \sum_{p=-N}^{N}\left\langle\overline{\widehat{G}\left(\mathbf{x}_{p}, \mathbf{y}, \omega\right)} \widehat{G}\left(\mathbf{x}_{p}, \mathbf{y}^{s}, \omega\right)\right\rangle,
$$

where $\widehat{G}$ is the random, time harmonic Green's function and where, because of the self-averaging property of $\Gamma^{T R}\left(\mathbf{y}^{s}, t\right)$ (see $[8,38]$ ), we can take the expectation of $\bar{G} \widehat{G}$. We evaluate the field at the exact range $(\eta=0)$ using the moment formula [32, 41],

$$
\left\langle\overline{\widehat{G}\left(\mathbf{x}_{p}, \mathbf{y}, \omega\right)} \widehat{G}\left(\mathbf{x}_{p}, \mathbf{y}^{s}, \omega\right)\right\rangle \approx \overline{\widehat{G}_{0}\left(\mathbf{x}_{p}, \mathbf{y}, \omega\right)} \widehat{G}_{0}\left(\mathbf{x}_{p}, \mathbf{y}^{s}, \omega\right) e^{-\frac{k^{2} \xi^{2} a_{e}^{2}}{2 L^{2}}}
$$

where

$$
a_{e}=a_{e}(L)=\sqrt{D L^{3}},
$$

is a length that defines the effective aperture and $D$ is a reciprocal length parameter that depends only on the statistics of the random fluctuations of the speed of propagation. This moment formula 
is valid in the regime of the paraxial and white noise approximation, which is discussed further in the appendix. Using this formula we have [8]

$$
\widehat{\Gamma}^{T R}(\xi, \eta=0, \omega) \approx \overline{\widehat{f}(\omega)} e^{-\frac{k^{2} \xi^{2} a_{e}^{2}}{2 L^{2}}} \sum_{p=-N}^{N} \overline{\widehat{G}_{0}\left(\mathbf{x}_{p}, \mathbf{y}, \omega\right)} \widehat{G}_{0}\left(\mathbf{x}_{p}, \mathbf{y}^{s}, \omega\right)=\widehat{\Gamma}_{0}^{T R}(\xi, \eta=0, \omega) e^{-\frac{k^{2} \xi^{2} a_{e}^{2}}{2 L^{2}}}
$$

and in time domain,

$$
\begin{aligned}
\Gamma^{T R}(\xi, \eta=0, t) & \approx \frac{c_{0}}{8 \pi^{2} L h \xi \sin \alpha} \frac{1}{\sqrt{2 \pi \sigma_{t}^{2}\left(1+\epsilon^{2}\right)}} e^{-i \frac{\omega_{0}}{1+\epsilon^{2}}\left(-t+\frac{\xi^{2}}{2 c_{0} L}\right)-\omega_{0}^{2} \frac{a_{e}^{2} \xi^{2}}{2 c_{0}^{2} L^{2}\left(1+\epsilon^{2}\right)} \times} \\
& {\left[e^{-i \omega_{0} \frac{\xi a \sin \alpha}{2 c_{0} L\left(1+\epsilon^{2}\right)}-\frac{\left(-t+\frac{\xi^{2}}{2 c_{0} L}+\frac{\xi a \sin \alpha}{2 c_{0} L}\right)^{2}}{2 \sigma_{t}^{2}\left(1+\epsilon^{2}\right)}}-e^{i \omega_{0} \frac{\xi a \sin \alpha}{2 c_{0} L\left(1+\epsilon^{2}\right)}-\frac{\left(-t+\frac{\xi^{2}}{2 c_{0} L}-\frac{\xi a \sin \alpha}{2 c_{0} L}\right)^{2}}{2 \sigma_{t}^{2}\left(1+\epsilon^{2}\right)}}\right], }
\end{aligned}
$$

where $\xi$ is assumed to vary over a region smaller than,

$$
\xi \ll \frac{\sigma_{t} c_{0} L}{a_{e}}=\frac{\omega_{0} \sigma_{t}}{2 \pi} \frac{\lambda_{0} L}{a_{e}}=\frac{1}{B} \frac{\lambda_{0} L}{a_{e}},
$$

so that $\epsilon=\frac{a_{e} \xi}{\sigma_{t} c_{0} L} \ll 1$. Since the pulse is short, we evaluate the right hand side of (3.15) at the arrival time $t=\frac{\xi^{2}}{2 c_{0} L}$ and we get,

$$
\Gamma^{T R}\left(\xi, 0, \frac{\xi^{2}}{2 c_{0} L}\right) \approx \frac{c_{0}}{4 \pi^{2} L h \xi} \frac{-i}{\sqrt{2 \pi \sigma_{t}^{2}}} \sin \left(\frac{\omega_{0} \xi a}{2 c_{0} L}\right) e^{-\frac{A_{A^{2}}^{2} \xi^{2}}{8 c_{0}^{2} L^{2} \sigma_{t}^{2}}}=\Gamma_{0}^{T R}\left(\xi, 0, \frac{\xi^{2}}{2 c_{0} L}\right) e^{-\frac{2 \pi^{2} a_{e}^{2}}{L^{2} \lambda_{0}^{2}} \xi^{2}} .
$$

Here $A_{e}$ is the broad-band effective aperture given by

$$
A_{e}^{2}=a^{2}+4 \omega_{0}^{2} \sigma_{t}^{2} a_{e}^{2}=a^{2}+\left(\frac{4 \pi a_{e}}{B}\right)^{2} .
$$

We can also write $(3.17)$ in the form

$$
\Gamma^{T R}\left(\xi, 0, \frac{\xi^{2}}{2 c_{0} L}\right) \approx \frac{c_{0}}{4 \pi^{2} L h \xi} \frac{-i}{\sqrt{2 \pi \sigma_{t}^{2}}} \sin \left(\frac{\omega_{0} \xi a}{2 c_{0} L}\right) e^{-\frac{\xi^{2}}{2 s_{R}^{2}}}
$$

where the variance of the Gaussian $s_{R}$ is given by

$$
s_{R}=\frac{2}{B} \frac{\lambda_{0} L}{A_{e}} .
$$

This is just like (3.9) but with the physical aperture $a$ replaced by the effective aperture $A_{e}$. From (3.18) we note that the product $B A_{e}$ is

$$
B A_{e}=\sqrt{B^{2} a^{2}+\left(4 \pi a_{e}\right)^{2}},
$$

so that for narrow-band signals the physical aperture $a$ is negligible while for broad-band ones it may contribute to super-resolution. By super-resolution we mean the ordering $a<A_{e}$. We often have $a \ll A_{e}$ when there is substantial multipathing. 
In the numerical simulations the range $L$ is limited by our computational capabilities so $a_{e}$ is roughly the same as $a$ and the physical aperture as well as the bandwidth play a role in (3.21). We also see from (3.21) that super-resolution is enhanced by larger bandwidths, as is the statistical stability in time reversal refocusing. Because $B$ is the relative bandwidth, $0<B<1$, the value of $B$ plays a significant role when $a$ and $a_{e}$ are of the same order but does not affect super-resolution if $a \ll a_{e}$. However, narrow bandwidth will affect the statistics. At fixed frequency, $\widehat{\Gamma}^{T R}$ is a random quantity which may exhibit or not focusing, depending on the realization of the medium [8]. In a broadband regime, due to the frequency diversity and the decorrelation of $\widehat{\Gamma}^{T R}\left(\mathbf{y}^{s}, \omega\right)$, for different frequencies $[8,39]$, we obtain by Fourier synthesis a stable quantity $\Gamma^{T R}\left(\mathbf{y}^{s}, t\right)$. The precise bandwidth needed to ensure statistical stability is not known but we can use the following empirical rule: If $\Delta \omega$ is an estimate of the decoherence frequency interval for $\widehat{\Gamma}^{T R}$, then the bandwidth $B$ divided by $\Delta \omega$ should be large $(\sim 30-50)$.

\section{Interferometric and matched field imaging}

In time reversal, one does not need to know the source location, but in imaging, one wishes to find it, from the received echoes at the array. While it is often said that all imaging methods use time reversal, there is a significant difference. Real time reversal is done by sending the recorded, reversed signals at the array, back into the same random medium while in imaging the back propagation is done analytically or numerically, in a reference medium. For example, when imaging in random media with index of refraction (2.5), we do not know the fluctuations of the sound speed, so we use a reference, homogeneous medium with sound speed $c_{0}$. The imaging point spread function is given by (2.10) and it is radically different from $\Gamma^{T R}$, in random media. First, unlike $\Gamma^{T R}, \Gamma^{I M}$ is wider in random media than in homogeneous ones, because multiple scattering impedes the identification of the source location. Second, the imaging point-spread function is not self-averaging, which is a serious problem that must be addressed through appropriate data processing.

A key point in the self-averaging of $\Gamma^{T R}$ is the approximate cancellation of phases of the random Green's functions between $\mathbf{y}$ and the array, and between the array and $\mathbf{y}^{s}$, respectively. Such phase cancellations do not occur in (2.10). Our key observation is that we can achieve phase cancellations by taking correlations of the recorded signals at the array. For example, in the remote regime with $a \ll L$, by taking the autocorrelation of $\Gamma^{I M}$, we obtain a self-averaging functional. At zero time-lag this is the matched field imaging functional

$$
\Gamma^{M F}\left(\mathbf{y}^{s}\right)=\int_{-\infty}^{\infty}\left|\widehat{\Gamma}^{I M}\left(\mathbf{y}^{s}, \omega\right)\right|^{2} d \omega
$$

which is statistically stable and provides an estimate of the cross-range of the source. The range is lost, however, and it must be estimated separately, from arrival time information, for example.

The matched field functional can be given an interferometric interpretation as follows. Introduce the trace autocorrelations or interferograms at the array

$$
S_{p q}^{I M}=s_{p}(\cdot) * s_{q}(-\cdot)\left(t_{p}\left(\mathbf{y}^{s}\right)-t_{q}\left(\mathbf{y}^{s}\right)\right)
$$

where $*$ denotes convolution in time. Note that the autocorrelation of the traces is evaluated at the difference between the travel time from $\mathbf{x}_{p}$ to $\mathbf{y}^{s}$ and $\mathbf{x}_{q}$ to $\mathbf{y}^{s}$ because a peak is expected when $\mathbf{y}^{s}$ is close to the source location $\mathbf{y}$. Now, if we sum these interferograms we get, up to scaling factors, the matched field functional. Thus,

$$
\Gamma^{K M F}\left(\mathbf{y}^{s}\right)=\sum_{p, q} S_{p q}^{I M}
$$


the Kirchhoff matched field functional, is essentially equivalent to $\Gamma^{M F}\left(\mathbf{y}^{s}\right)$, that is, they peak at the same point $\mathbf{y}$.

Various functionals for imaging the location of an active source and their relative performance are considered in $[6,12]$. Our main interest and result in this paper is an analytical formula for the matched field functional $\Gamma^{M F}\left(\mathbf{y}^{s}\right)$ that can be used to estimate the narrow-band effective aperture $a_{e}$, when the range $L$ is known. The estimation of $a_{e}$ is important in assessing quantitatively super-resolution in time reversal and in estimating the loss of resolution of matched field imaging in random media.

\subsection{Matched field in homogeneous media}

In homogeneous media, the matched field estimator is

$$
\Gamma_{0}^{M F}(\xi, \eta)=\frac{1}{2 \pi} \int d \omega\left|\widehat{\Gamma}_{0}^{T R}(\xi, \eta, \omega)\right|^{2}
$$

Using now expression (3.6) and the Fourier coefficients of the pulse (2.2) in (4.4) we obtain,

$$
\Gamma_{0}^{M F}(\xi, \eta) \approx \frac{1}{2 \pi} \int d \omega\left(\frac{\omega}{8 \pi^{2} L^{2} h}\right)^{2} e^{-\sigma_{t}^{2}\left(\omega-\omega_{0}\right)^{2}}\left|\int_{-a / 2}^{a / 2} d x e^{-i \frac{\omega}{c_{0}}\left(\frac{\eta x^{2}}{2 L(L+\eta)}+\frac{x \xi}{L+\eta}\right)}\right|^{2}
$$

We show in Figure 2, the plot of $\Gamma_{0}^{M F}$ for the parameters in the numerical simulations described in section 5. Because $\Gamma_{0}^{M F}$ is an autocorrelation, range information is lost but the cross-range can be estimated, with a resolution that decreases as we search deeper in range (i.e. for large $\eta$ ). To illustrate the dependence of the cross-range resolution on the array aperture $a$, we evaluate (4.5) at the exact range $(\eta=0)$,

$$
\Gamma_{0}^{M F}(\xi, 0) \approx \frac{c_{0}^{2}}{64 \pi^{4} L^{2} h^{2} \sqrt{\pi \sigma_{t}^{2}}}\left[\frac{1-e^{-\frac{a^{2} \xi^{2}}{4 c_{0}^{2} L^{2} \sigma_{t}^{2}}}}{\xi^{2}}+2 \frac{\sin ^{2}\left(\frac{\omega_{0} \xi a}{2 c_{0} L}\right)}{\xi^{2}} e^{-\frac{a^{2} \xi^{2}}{4 c_{0}^{2} L^{2} \sigma_{t}^{2}}}\right]
$$

We note now that, as in time reversal in homogeneous media, the cross-range resolution of the
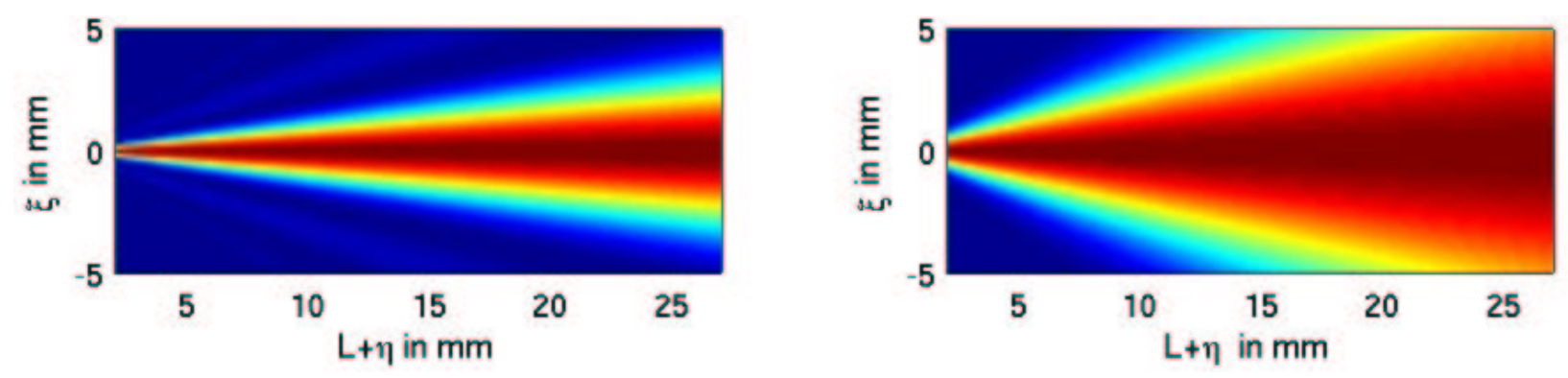

Figure 2: The matched field estimator in deterministic media (left figure, equation (4.6)) and in random media (right figure, equation (4.16)), for an effective aperture $a_{e}=2 a$.

matched field estimator is inversely proportional to the aperture $a$. In the next section, we show that in random media, the matched field functional behaves very differently than time reversal. In particular, we show that because of multiple scattering by the inhomogeneities, the images are blurred in a manner quantified by the narrow-band effective aperture $a_{e}$. 


\subsection{Matched field in random media}

The matched field functional in random media is

$$
\widehat{\Gamma}^{M F}\left(\mathbf{y}^{s}, \omega\right)=|\widehat{f}(\omega)|^{2} \sum_{p=-N}^{N} \sum_{q=-N}^{N} \overline{\widehat{G}_{0}\left(\mathbf{x}_{p}, \mathbf{y}^{s}, \omega\right)} G\left(\mathbf{x}_{p}, \mathbf{y}, \omega\right) \overline{\widehat{G}\left(\mathbf{x}_{q}, \mathbf{y}, \omega\right)} G_{0}\left(\mathbf{x}_{q}, \mathbf{y}^{s}, \omega\right) .
$$

Because of its statistical stability in the time domain $[8,39], \int d \omega \widehat{\Gamma}^{M F}\left(\mathbf{y}^{s}, \omega\right)$ is essentially equal to its expectation. Using the moment formula (see $[32,41]$ and the appendix),

$$
\left\langle\overline{\widehat{G}\left(\mathbf{x}_{p}, \mathbf{y}, \omega\right)} \widehat{G}\left(\mathbf{x}_{q}, \mathbf{y}, \omega\right)\right\rangle \approx \overline{\widehat{G}_{0}\left(\mathbf{x}_{p}, \mathbf{y}, \omega\right)} \widehat{G}_{0}\left(\mathbf{x}_{q}, \mathbf{y}, \omega\right) e^{-\frac{k^{2} a_{e}^{2}}{2 L^{2}}\left|\mathbf{x}_{p}-\mathbf{x}_{q}\right|^{2}},
$$

and equations (3.3), (3.4) we obtain

$$
\widehat{\Gamma}^{M F}(\xi, \eta, \omega) \approx \frac{|\widehat{f}(\omega)|^{2}}{(4 \pi L)^{4}} \sum_{p=-N}^{N} \sum_{q=-N}^{N} e^{-\frac{k^{2} a_{e}^{2}}{2 L^{2}}\left(x_{p}-x_{q}\right)^{2}+i k\left(x_{p}-x_{q}\right)\left(\frac{\left(x_{p}+x_{q}\right) \eta}{2 L(L+\eta)}+\frac{\xi}{L+\eta}\right)} .
$$

As in section 3, we replace the sums in (4.9) with integrals and we let $x_{p}=p h / 2 \rightarrow x$ and $x_{q}=q h / 2 \rightarrow \tilde{x}$ so that $(4.9)$ becomes

$$
\widehat{\Gamma}^{M F}(\xi, \eta, \omega) \approx \frac{4|\widehat{f}(\omega)|^{2}}{(4 \pi L)^{4} h^{2}} \int_{-a / 2}^{a / 2} d x \int_{-a / 2}^{a / 2} d \tilde{x} e^{-\frac{k^{2} a_{e}^{2}}{2 L^{2}}(x-\tilde{x})^{2}+i k(x-\tilde{x})\left(\frac{(x+\tilde{x}) \eta}{2 L(L+\eta)}+\frac{\xi}{L+\eta}\right)} .
$$

Now, let us make the change of variables

$$
u=x-\tilde{x}, v=\frac{x+\tilde{x}}{2},
$$

which maps the square $[-a / 2, a / 2] \times[-a / 2, a / 2]$ into a parallelogram. Clearly, $v \in[-a / 2, a / 2]$ and, since the integrand in (4.10) decays in $u$ as $e^{-\frac{k^{2} a_{e}^{2} u^{2}}{2 L^{2}}}$, only the vicinity of $u=0$ matters. More precisely, we have a Gaussian in $u$ with variance $\sigma^{2}$,

$$
\sigma^{2}=\frac{L^{2}}{k^{2} a_{e}^{2}}=\frac{L^{2} \lambda^{2}}{4 \pi^{2} a_{e}^{2}}
$$

which is small because it is proportional to the refocusing spot size in time reversal. We can therefore integrate over all $u$ on the real line

$$
\widehat{\Gamma}^{M F}(\xi, \eta, \omega) \approx \frac{4|\widehat{f}(\omega)|^{2}}{(4 \pi L)^{4} h^{2}} \int_{-a / 2}^{a / 2} d v e^{-\frac{k^{2} \sigma^{2}}{2}\left(\frac{\eta v}{L(L+\eta)}+\frac{\xi}{L+\eta}\right)^{2}} \int_{-\infty}^{\infty} d u e^{-\frac{1}{2 \sigma^{2}}\left[u-i k \sigma^{2}\left(\frac{v \eta}{L(L+\eta)}+\frac{\xi}{L+\eta}\right)\right]^{2}} .
$$

The integral in $u$ is equal to $\sqrt{2 \pi} \sigma$. The variance of the $v$ integrand is large compared to $a$,

$$
\frac{L^{2}(L+\eta)^{2}}{a^{2} k^{2} \sigma^{2} \eta^{2}}=\left(\frac{L+\eta}{\eta}\right)^{2}\left(\frac{a_{e}}{a}\right)^{2} \gg 1
$$

at least for small $\eta$ and/or $a_{e} \gg a$. We can thus approximate the integral in $v$ by the value of the integrand at $v=0$, times the aperture $a$. The result is

$$
\widehat{\Gamma}^{M F}(\xi, \eta, \omega) \approx C(L, a, \lambda) e^{-\frac{\xi^{2}}{2(L+\eta)^{2}}\left(\frac{L}{a_{e}}\right)^{2}},
$$


where

$$
C(L, a, \lambda)=\frac{4 a L \lambda|\widehat{f}(\omega)|^{2}}{\sqrt{2 \pi} h^{2} a_{e}(4 \pi L)^{4}},
$$

is independent of the search point $\mathbf{y}^{s}$. We see from (4.14) that the zero-lag matched field functional is essentially independent of frequency in random media, so that summing over frequencies we have

$$
\Gamma^{M F}(\xi, \eta) \approx \tilde{C}(L, a) e^{-\frac{\xi^{2}}{2(L+\eta)^{2}}\left(\frac{L}{a_{e}}\right)^{2}}
$$

where $\tilde{C}(L, a)$ is given by

$$
\tilde{C}(L, a)=\int_{-\infty}^{\infty} C\left(L, a, \lambda=\frac{2 \pi c_{0}}{\omega}\right) d \omega
$$

and is independent of the cross-range $\xi$.

We have now a simple analytical expression (4.16) that allows us to assess the effect of the random medium when imaging an active source by the matched field functional. We must know the range $L$ of the target, however, since it is the ratio $a_{e} / L$ that appears in (4.16). Unlike time reversal, where a larger $a_{e}$ (i.e. stronger multipath) gives a tighter point-spread function (see equation (3.17)), in imaging the resolution is worse with larger $a_{e}$. Rich scattering environments produce blurry images of active stationary (not moving) sources. As we will show in section 6, this is true in general for imaging in random media.

\section{Estimation of the effective aperture}

Using the explicit dependence of $\Gamma^{M F}$ on $a_{e}$, we estimate the effective aperture by matching the formula (4.16) with the numerically calculated imaging functional $\Gamma_{n}^{M F}(\xi, \eta)$. The setup of the numerical simulations is described below.

\subsection{Setup for the numerical simulations}

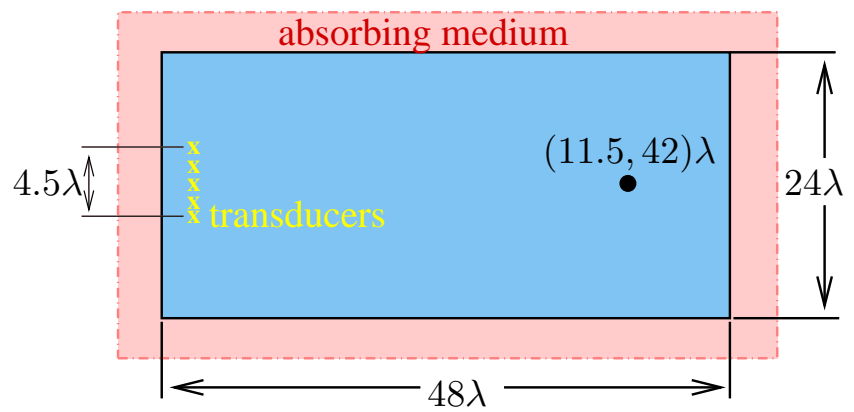

Figure 3: The computational setup. The dimensions of the problem are given in terms of the central wavelength $\lambda=0.5 \mathrm{~mm}$. The medium is considered to be infinite in all directions so in the numerical computations an absorbing layer surrounds the domain.

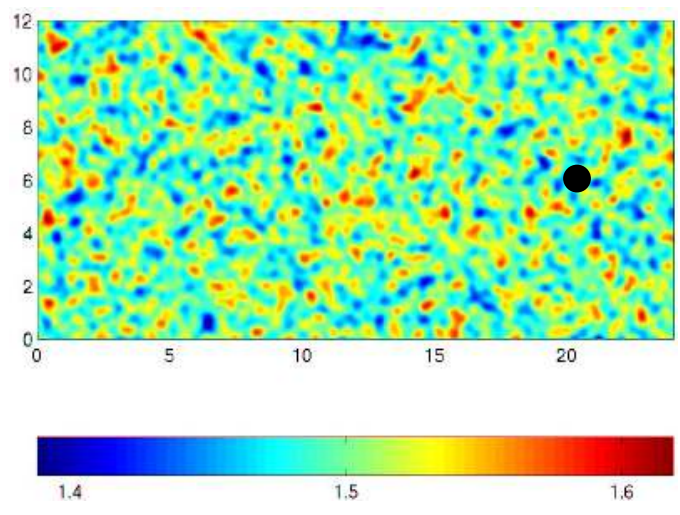

Figure 4: A typical realization of the random sound speed $c(\mathbf{x})$. The target is shown as a large black dot •. The units in the horizontal and vertical axes are $\mathrm{mm}$ and, in the color bar, $\mathrm{km} / \mathrm{s}$. The standard deviation for this example is $s=$ $4.95 \%$ 
We take an array of 10 transducers at a distance $h=\lambda_{0} / 2$ from each other, which means that $a=2.25 \mathrm{~mm}$. The active source is a square of size $\lambda_{0} / 30$, located at range $L=2 \mathrm{~cm}$ and at zero cross-range, measured with respect to the center of the array. The pulse (2.1) has width $\sigma_{t}=0.2325 \mu \mathrm{s}$, central frequency $2 \pi \omega_{0}=3 \mathrm{MHz}$ (i.e. $\lambda_{0}=0.5 \mathrm{~mm}$ ) and the bandwidth is $2-4 \mathrm{MHz}$ (measured at $6 \mathrm{~dB})$. The field $c(\mathbf{x})$ is generated as a random Fourier series with mean $c_{0}=1.5 \mathrm{~km} / \mathrm{s}$ and Gaussian correlation, with correlation length $l=0.3 \mathrm{~mm}$. The standard deviation ranges from $1 \%$ to $5 \%$. An example of a realization of the random medium is shown in Figure 4. We calculate the acoustic pressure at the array by solving the wave equation in the time domain, with a finite element method that discretizes the mixed velocity-pressure (first order system) formulation [3, 4]. To simulate the infinite medium, we surround the computational domain by a perfectly matched absorbing layer (cf. [5]), as shown in Figure 3.

All computations are done in two dimensions, to avoid excessive computational time but the analysis is based on three dimensional Green's functions. However, we have seen that, in remote sensing regimes, it is the phase of the Green's function and not its amplitude that matters. Since the phases of the Green's functions are the same in two and in three dimensions, we expect that the results of our direct numerical computations will be in good agreement with the theory.

We note finally that the numerical simulations are done with solutions of the wave equation and not its parabolic approximation, which is used in deriving the theoretical results. The ratio $a / L \approx .125$ here.

\subsection{The estimation of $a_{e}$}

To find $a_{e}$, we match formula (4.16) with the numerically computed $\Gamma_{n}^{M F}(\xi, \eta)$ as follows. We calculate numerically the signals $s_{p}(t)$ received at the array elements $p=-N, \ldots, N$, for a time interval of length $T$, which is long enough to ensure that all is quiet for $t>T$. The numerical matched field functional at the search point

$$
\mathbf{y}^{s}=(\xi, 0, L+\eta)
$$

is

$$
\Gamma_{n}^{M F}(\xi, \eta)=\int \widehat{\Gamma_{n}^{M F}}(\xi, \eta, \omega) d \omega
$$

where

$$
\widehat{\Gamma_{n}^{M F}}(\xi, \eta, \omega)=\left|\sum_{p=1}^{N} \overline{\widehat{s_{p}}(\omega)} \widehat{G}_{0}\left(\mathbf{x}_{p}, \mathbf{y}^{s}, \omega\right)\right|^{2} .
$$

To find $a_{e}$ we sample the search domain in steps of $\lambda_{0} / 2$, and we minimize the discrepancy between the theoretical and numerical matched field functionals (4.16) and (5.1), respectively,

$$
\min _{\beta} \sum_{j=-5}^{5} \sum_{m=-4}^{4}\left[\gamma\left(j \frac{\lambda_{0}}{2}, m \frac{\lambda_{0}}{2}\right)-e^{-\frac{\beta\left(j \lambda_{0} / 2\right)^{2}}{2\left(L+m \lambda_{0} / 2\right)^{2}}}\right]^{2} .
$$

Here

$$
\beta=\left(\frac{L}{a_{e}}\right)^{2},
$$

and for each $\eta$ we normalize (5.1) by

$$
\gamma(\xi, \eta)=\Gamma_{n}^{M F}(\xi, \eta) / \max _{\xi} \Gamma_{n}^{M F}(\xi, \eta) .
$$


The minimization (5.3) is done in MATLAB with fminunc. We find $a_{e}$ from the minimizer $\beta$, by substituting the correct range $L$ in (5.4).

To assess the feasibility and robustness of our estimation approach, we take different realizations of the random medium. Since $\Gamma^{M F}$ is self-averaging, we expect the value of the estimated $a_{e}$ to be stable. Take for example four realizations of the random medium. The estimated $a_{e}$ are $1.43,1.54,1.37,1.46$ in $\mathrm{mm}$, respectively, and the fluctuations are small (the standard deviation about the mean 1.45 is 7\%). We show in the top two panels of Figure 5 the numerically computed matched field functional (5.1), for two realizations. The theoretical matched field functional (4.16), when calculated with the estimated $a_{e}$ looks the same as the numerical ones.

Once $a_{e}$ is estimated, we can use it to compute the time reversal functional $\Gamma^{T R}$ given by (3.19). This prediction can be compared with the numerically computed time reversal point spread function, in order to assess the feasibility of our estimation approach. The results are shown in the bottom panels of Figure 5. Note the super-resolution phenomenon, manifested by the tighter focal spot in the random medium than in the homogeneous one. Note also that the theoretical formula (3.17) with the estimated $a_{e}$ gives a rather accurate spot size at the source. The fluctuations that we observe (for large $\xi$ )in the numerically computed field (right bottom panels) are not captured by the theoretical result (3.17), as expected. However, near the source, the refocused field is predicted accurately. The statistical stability of both (5.1) and the time reversed field is observed in numerical simulations with several realizations of the random medium.

\section{Application to distributed reflectivity imaging}

We will consider briefly the inverse problem of imaging the reflectivity $\varrho(\mathbf{y})$ of an extended target compactly supported in the domain $\mathcal{D} \subset \mathbb{R}^{3}$. As in the previous section, our objective here is to analyze the resolution obtained by the matched field imaging method when the target is embedded in a randomly inhomogeneous medium. The regime of physical scales is the same as previously, i.e., we focus attention on remote sensing in a random medium with significant multipathing and a homogeneous background. The geometry of the problem is shown in Figure 6.

Signals are recorded on two small linear arrays covering a possibly large synthetic aperture. The physical aperture of the transmitting array is $a_{s}$ and that of the receiving one $a_{r}$. Let us assume that a reference point of $\mathcal{D}$ is located at

$$
\mathbf{y}_{0}=(0,0, L)
$$

with respect to the center $\mathcal{O}_{s}$ of the transmitter array. Both arrays have point transducers located on the $\mathrm{x}$-axis and separated by $h / 2$, as in section 2 . That is, we assume that the array of transmitters is orthogonal to the axis $\mathcal{O}_{s} \mathbf{y}_{0}, \mathcal{O}_{s}$ being the origin of our coordinate system, and we denote by $\alpha$ the angle between $\mathcal{O}_{s} \mathbf{y}_{0}$ and $\mathcal{O}_{r} \mathbf{y}_{0}$ (see Figure 6). The transmitter and receiver array elements are located at

$$
\begin{array}{ll}
\text { transmitter: } & \mathbf{x}_{p}=\left(x_{p}, 0,0\right), x_{p}=p \frac{h}{2}, p=-N, \ldots, N, \\
\text { receiver: } & \mathbf{x}_{r}=\left(\mathcal{O}_{r}+x_{r}, 0,0\right), x_{r}=r \frac{h}{2}, r=-N_{r}, \ldots, N_{r} .
\end{array}
$$

The data for the distributed reflectivity imaging problem is the response matrix $P\left(\mathbf{x}_{p}, \mathbf{x}_{r}, t\right)$, obtained as follows. A pulse $f(t)$ is emitted from an array element, located at $\mathbf{x}_{p}$, and the backscattered returns, $P\left(\mathbf{x}_{p}, \mathbf{x}_{r}, t\right)$, are recorded at $\mathbf{x}_{r}, r=-N_{r}, \ldots, N_{r}$, for a sufficiently long time interval $[0, T]$. Probing of the medium is done by using all transducers at $\mathbf{x}_{p}, p=-N, \ldots, N$. 

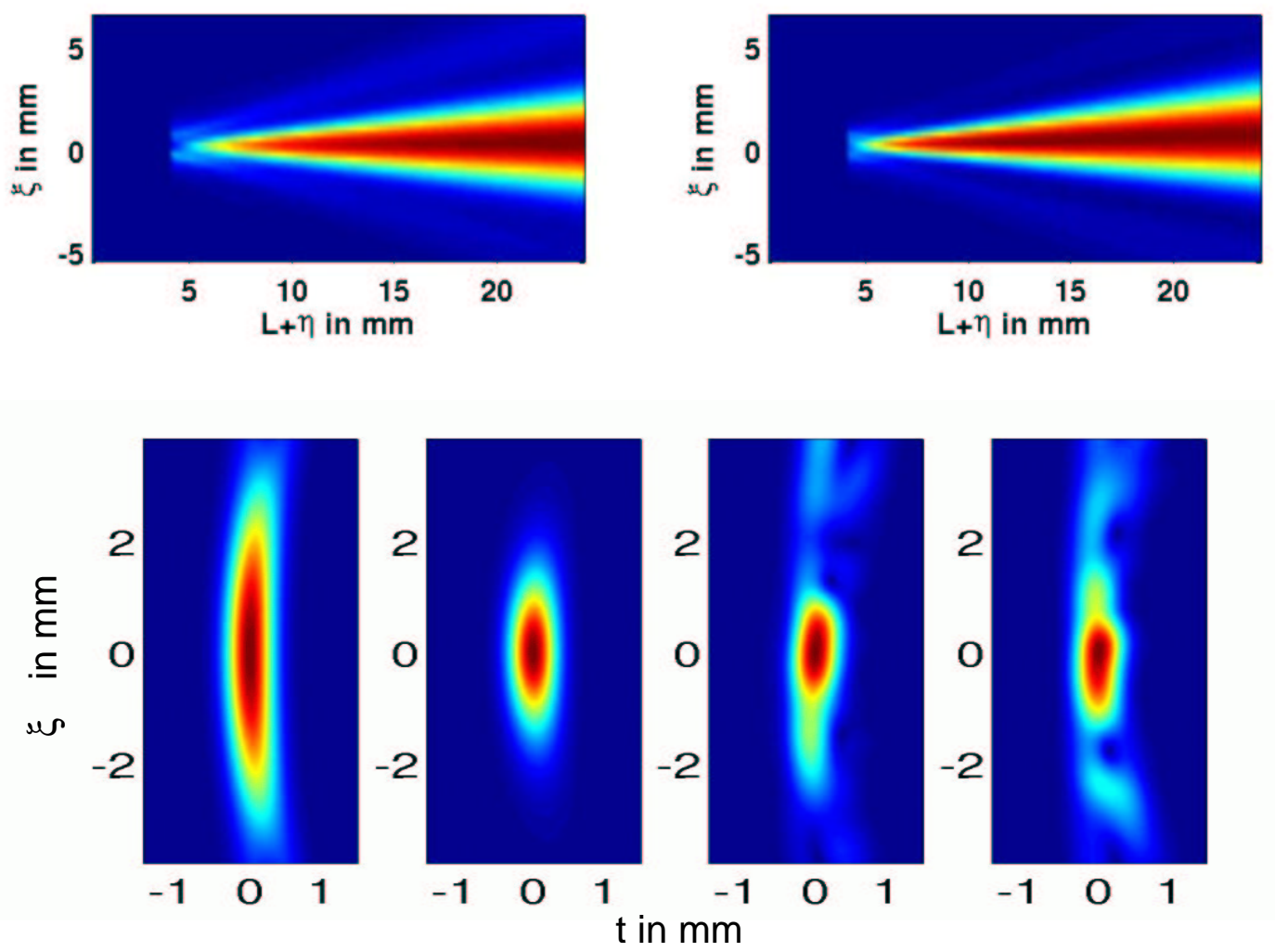

Figure 5: Top: $\Gamma^{\mathrm{MF}}\left(\mathbf{y}^{s}\right)$ computed numerically for two realizations of the random medium, the estimation of $a_{e}$ is $1.43 \mathrm{~mm}$ (resp. $1.47 \mathrm{~mm}$ ) for the data on the left (resp. right). The horizontal axis is $L+\eta$ in $\mathrm{mm}$ and the vertical axis is the cross-range $\xi$ in mm. Bottom (left to right): the theoretical prediction of the time reversed field in a homogeneous medium, in a random medium (obtained using $a_{e}=1.45 \mathrm{~mm}$ in (3.17)) and the numerically calculated time reversed field for the same two realizations of the random medium considered in the top matched field estimates. The horizontal axis is time in $\mu$ s and the vertical axis is the cross-range $\xi$ in $\mathrm{mm}$.

To analyze the resolution of the matched field imaging method for this extended reflectivity problem, we first introduce an imaging functional which is the generalization of the method presented in section 4 . We consider here a more general setup (see Figure 6) with a source and a receiver array offset by an angle $\alpha$. Note that, the geometry of the previous sections can be recovered by letting $\alpha=0$. The overall range $L$ of the extended reflector is assumed fixed and, because we are interested in a remote sensing regime, we also assume that

$$
\frac{a_{s}}{L} \ll 1, \frac{a_{r}}{L} \ll 1 \text { and } \frac{\left|\mathbf{y}-\mathbf{y}_{0}\right|}{L} \ll 1, \forall \mathbf{y} \in \mathcal{D} .
$$

Using the Born approximation for scattering from the extended reflector we have the following theoretical expression for the response matrix

$$
P\left(\mathbf{x}_{p}, \mathbf{x}_{r}, t\right)=\frac{1}{2 \pi} \int_{-\infty}^{\infty} e^{-i \omega t} \widehat{P}\left(\mathbf{x}_{p}, \mathbf{x}_{r}, \omega\right) d \omega
$$




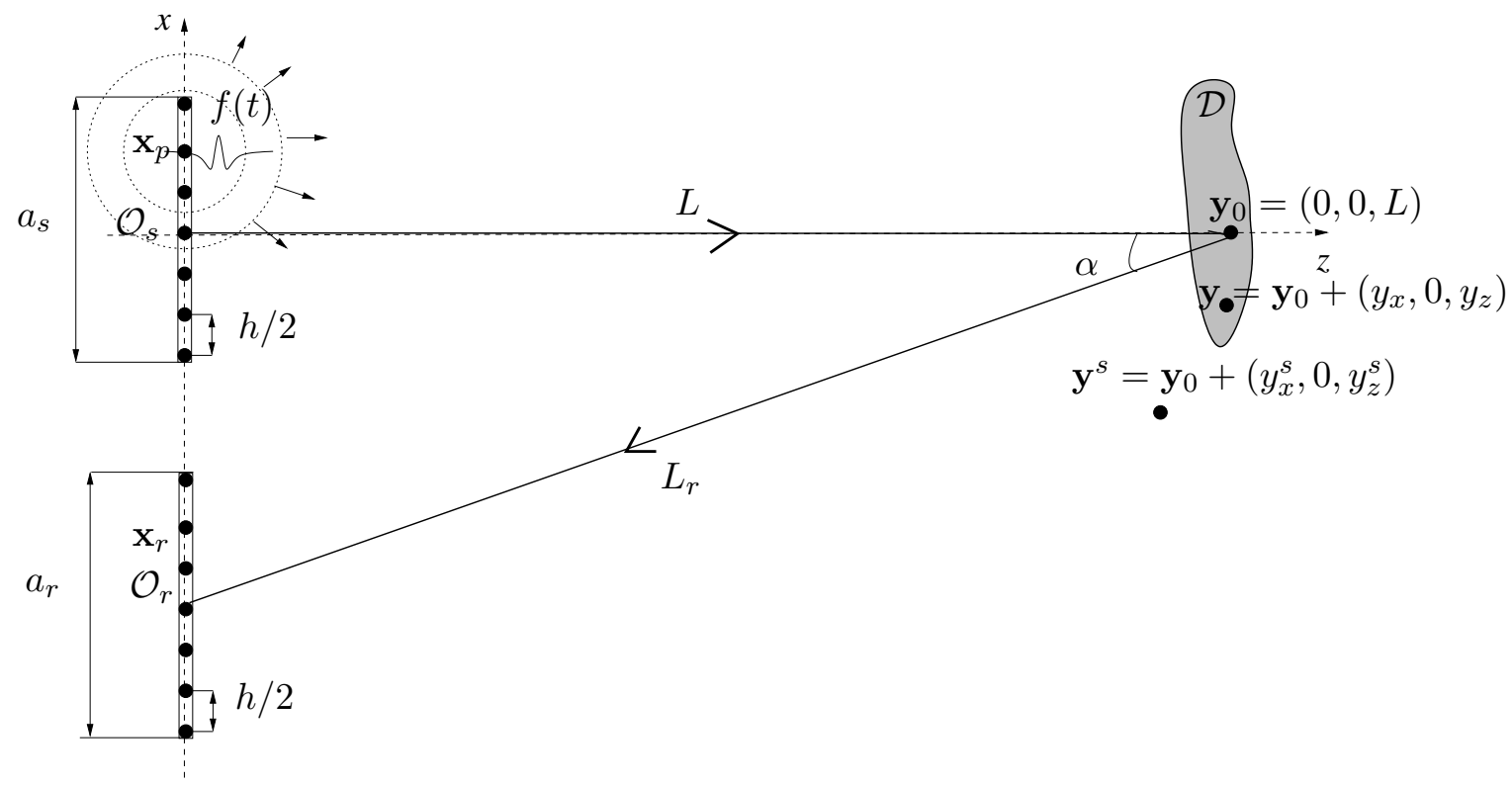

Figure 6: The setup for distributed reflectivity imaging.

with,

$$
\widehat{P}\left(\mathbf{x}_{p}, \mathbf{x}_{r}, \omega\right)=k^{2} \widehat{f}(\omega) \int_{\mathcal{D}} \varrho(\mathbf{y}) \widehat{G}\left(\mathbf{x}_{p}, \mathbf{y}, \omega\right) \widehat{G}\left(\mathbf{x}_{r}, \mathbf{y}, \omega\right) d \mathbf{y}
$$

Here, as before, $k=\omega / c_{0}$ is the wavenumber, $c_{0}$ is a reference speed of propagation and $\widehat{G}\left(\mathbf{x}_{p}, \mathbf{y}, \omega\right)$ is the two point (random) Green's function satisfying the reduced wave equation (2.4). We note that the data, i.e, the elements of the response matrix, are not self-averaging because the product of the random Green's functions $\widehat{G}\left(\mathbf{x}_{p}, \mathbf{y}, \omega\right) \widehat{G}\left(\mathbf{x}_{r}, \mathbf{y}, \omega\right)$ has a large random phase, for there is no complex conjugation (time reversal) in (6.7) to eliminate it.

To image the unknown reflectivity $\varrho(\mathbf{y})$ we back-propagate each element of the response matrix $\widehat{P}\left(\mathbf{x}_{p}, \mathbf{x}_{r}, \omega\right)$ to the search point $\mathbf{y}^{s}$ in a homogeneous medium. This can be done by evaluating $P\left(\mathbf{x}_{p}, \mathbf{x}_{r}, t\right)$ at the deterministic arrival time

$$
t_{p r}\left(\mathbf{y}^{s}\right)=\frac{\left|\mathbf{x}_{p}-\mathbf{y}^{s}\right|}{c_{0}}+\frac{\left|\mathbf{x}_{r}-\mathbf{y}^{s}\right|}{c_{0}}
$$

which gives the Kirchhoff migration [7, 17] imaging functional

$$
\mathcal{S}^{I M}\left(\mathbf{x}_{p}, \mathbf{x}_{r}, \mathbf{y}^{s}\right)=P\left(\mathbf{x}_{p}, \mathbf{x}_{r}, t=t_{p r}\left(\mathbf{y}^{s}\right)\right)=\frac{1}{2 \pi} \int_{-\infty}^{\infty} \widehat{P}\left(\mathbf{x}_{p}, \mathbf{x}_{r}, \omega\right) e^{-i \omega t_{p r}\left(\mathbf{y}^{s}\right)} d \omega .
$$

As in the case of a point scatterer, (6.9) is not self-averaging. To obtain a statistically stable imaging functional we correlate $\mathcal{S}^{I M}\left(\mathbf{x}_{p}, \mathbf{x}_{r}, \mathbf{y}^{s}\right)$ with the time-reversed $\mathcal{S}^{I M}\left(\mathbf{x}_{p}^{\prime}, \mathbf{x}_{r}^{\prime}, \mathbf{y}^{s}\right)$, that is, we multiply them and we sum over the arrays elements $x_{p}, x_{r}, x_{p}^{\prime}, x_{r}^{\prime}$, which gives

$$
\begin{aligned}
& \Gamma^{I M}\left(\mathcal{O}_{r}, \mathcal{O}_{s}, \mathbf{y}^{s}\right)= \\
& \sum_{p=-N}^{N} \sum_{p^{\prime}=-N}^{N} \sum_{r=-N_{r}}^{N_{r}} \sum_{r^{\prime}=-N_{r}}^{N_{r}} \frac{1}{2 \pi} \int_{-\infty}^{\infty} \widehat{P}\left(\mathbf{x}_{p}, \mathbf{x}_{r}, \omega\right) \widehat{P}\left(\mathbf{x}_{p}^{\prime}, \mathbf{x}_{r}^{\prime}, \omega\right) e^{-i \omega\left(t_{p r}\left(\mathbf{y}^{s}\right)-t_{p^{\prime} r^{\prime}}\left(\mathbf{y}^{s}\right)\right)} d \omega .
\end{aligned}
$$


Since $h$ is small compared to $a_{s}, a_{r}$ we can approximate the sum by an integral as in section 4 ,

$$
\begin{array}{r}
\Gamma^{I M}\left(\mathcal{O}_{r}, \mathcal{O}_{s}, \mathbf{y}^{s}\right)=\left(\frac{2}{h}\right)^{4} \int_{-a_{s} / 2}^{a_{s} / 2} \int_{-a_{s} / 2}^{a_{s} / 2} \int_{-a_{r} / 2}^{a_{r} / 2} \int_{-a_{r} / 2}^{a_{r} / 2} d x_{p} d x_{p}^{\prime} d x_{r} d x_{r}^{\prime} \\
\cdot \frac{1}{2 \pi} \int_{-\infty}^{\infty} d \omega \widehat{P}\left(\mathbf{x}_{p}, \mathbf{x}_{r}, \omega\right) \widehat{P}\left(\mathbf{x}_{p}^{\prime}, \mathbf{x}_{r}^{\prime}, \omega\right) e^{-i \omega\left(t_{p r}\left(\mathbf{y}^{s}\right)-t_{p^{\prime} r^{\prime}}\left(\mathbf{y}^{s}\right)\right)}
\end{array}
$$

In the following we will systematically use (6.11) with the integrals instead of (6.10). In more detail, the Kirchhoff migration imaging functional for the distributed reflectivity is

$$
\begin{aligned}
\Gamma^{I M}\left(\mathcal{O}_{r}, \mathcal{O}_{s}, \mathbf{y}^{s}\right)=\left(\frac{2}{h}\right)^{4} \int_{-a_{s} / 2}^{a_{s} / 2} \int_{-a_{s} / 2}^{a_{s} / 2} \int_{-a_{r} / 2}^{a_{r} / 2} \int_{-a_{r} / 2}^{a_{r} / 2} d x_{p} d x_{p}^{\prime} d x_{r} d x_{r}^{\prime} \\
\cdot \frac{1}{2 \pi} \int_{-\infty}^{\infty} d \omega\left(\frac{\omega}{c_{0}}\right)^{4}|\widehat{f}(\omega)|^{2} e^{-i \omega\left(t_{p r}\left(\mathbf{y}^{s}\right)-t_{p^{\prime} r^{\prime}}\left(\mathbf{y}^{s}\right)\right)} \\
\cdot \int_{\mathcal{D}} \int_{\mathcal{D}} d \mathbf{y} d \mathbf{y}^{\prime} \varrho(\mathbf{y}) \varrho\left(\mathbf{y}^{\prime}\right) \widehat{G}\left(\mathbf{x}_{p}, \mathbf{y}, \omega\right) \widehat{G}\left(\mathbf{x}_{r}, \mathbf{y}, \omega\right) \overline{\widehat{G}\left(\mathbf{x}_{p}^{\prime}, \mathbf{y}^{\prime}, \omega\right) \widehat{G}\left(\mathbf{x}_{r}^{\prime}, \mathbf{y}^{\prime}, \omega\right)}
\end{aligned}
$$

For this to be a self-averaging functional phase cancellation is needed, which means that $\mathbf{x}_{p}$ and $\mathbf{x}_{p}^{\prime}$ must be close to each other (resp. $\mathbf{x}_{r}$ and $\mathbf{x}_{r}^{\prime}$ ). This is why we consider the imaging functional $\Gamma^{I M}$ for small arrays. However, because we use an approximation like the one in (4.13), the array apertures $a_{s}$ and $a_{r}$ must be large compared to the spot size $\sigma$, defined in (4.12), but not too large so that this coarse graining uses too much of the data.

If we only have a single source rather than an array of sources, but still have an array of receivers, then we lose resolution in range, which we cannot recover by using data for different offsets $\alpha$ [11]. This seems surprising at first but it can be explained from the cone-like structure of the support of the matched field functional for point scatterers. We discuss this further in section 6.3.

\subsection{Imaging an active point scatterer}

Let us note briefly that this process of back-propagation, or Kirchhoff migration, and correlation is a generalization of the matched field method presented in section 4 . To see this, let us consider an active source located at $\mathbf{y}=(0,0, L)$ with the same geometry as above but with the receiver array at the location of the transmitter array, which is absent here. The receiver array elements are at $\mathbf{x}_{r}=\mathbf{x}_{p}=\left(x_{p}, 0,0\right), x_{p}=p h / 2, p=-N, \ldots, N$. The response matrix $P$ is now a vector and we can re-derive the matched field method in the following way.

We first back-propagate each element of the response vector $\widehat{P}\left(\mathbf{x}_{r}, \omega\right)$ to the search point $\mathbf{y}^{s}=$ $(\xi, 0, L+\eta)$ in a homogeneous medium. This can be done by evaluating $P\left(\mathbf{x}_{r}, t\right)$ at the deterministic arrival time,

We thus get,

$$
t_{r}\left(\mathbf{y}^{s}\right)=\frac{\left|\mathbf{x}_{r}-\mathbf{y}^{s}\right|}{c_{0}}
$$

$$
\mathcal{S}_{A}^{I M}\left(\mathbf{x}_{r}, \mathbf{y}^{s}\right)=P\left(\mathbf{x}_{r}, t=t_{r}\left(\mathbf{y}^{s}\right)\right)=\frac{1}{2 \pi} \int_{-\infty}^{\infty} \widehat{P}\left(\mathbf{x}_{r}, \omega\right) e^{-i \omega t_{r}\left(\mathbf{y}^{s}\right)} d \omega .
$$

To obtain a statistically stable imaging functional we consider the correlation of $\mathcal{S}_{A}^{I M}\left(\mathbf{x}_{r}, \mathbf{y}^{s}\right)$ with the time-reversed $\mathcal{S}_{A}^{I M}\left(\mathbf{x}_{r}^{\prime}, \mathbf{y}^{s}\right)$,

$$
\begin{aligned}
\frac{1}{2 \pi} \int_{-\infty}^{\infty} \widehat{P}\left(\mathbf{x}_{r}, \omega\right) \overline{\widehat{P}\left(\mathbf{x}_{r}^{\prime}, \omega\right)} e^{-i \omega\left(t_{r}\left(\mathbf{y}^{s}\right)-t_{r^{\prime}}\left(\mathbf{y}^{s}\right)\right)} d \omega= \\
\quad \frac{1}{2 \pi} \int_{-\infty}^{\infty}|\widehat{f}(\omega)|^{2} \widehat{G}\left(\mathbf{x}_{r}, \mathbf{y}, \omega\right) \overline{\widehat{G}\left(\mathbf{x}_{r}^{\prime}, \mathbf{y}, \omega\right)} e^{-i \omega\left(t_{r}\left(\mathbf{y}^{s}\right)-t_{r^{\prime}}\left(\mathbf{y}^{s}\right)\right)} d \omega
\end{aligned}
$$


Summing now (6.15) over the array elements $x_{r}, x_{r}^{\prime}$ gives the matched field function (4.16) (up to a constant),

$$
\begin{aligned}
& \Gamma_{A}^{I M}\left(L, a_{r}, a_{e}^{r}, \mathbf{y}^{s}\right)=\left(\frac{2}{h}\right)^{2} \int_{-a_{r} / 2}^{a_{r} / 2} \int_{-a_{r} / 2}^{a_{r} / 2} d x_{r} d x_{r}^{\prime} \frac{1}{2 \pi} \int_{-\infty}^{\infty} d \omega \widehat{P}\left(\mathbf{x}_{r}, \omega\right) \overline{\widehat{P}\left(\mathbf{x}_{r}^{\prime}, \omega\right)} e^{-i \omega\left(t_{r}\left(\mathbf{y}^{s}\right)-t_{r^{\prime}}\left(\mathbf{y}^{s}\right)\right)} \\
& \approx \tilde{C}^{\prime}\left(L_{r}, a_{r}\right) e^{-\frac{\xi^{2}}{2\left(L_{r}+\eta\right)^{2}}\left(\frac{L_{r}}{a_{e}^{r}}\right)^{2}} \text {. }
\end{aligned}
$$

If we identify $a_{r}=a, L_{r}=L$ and $a_{e}^{r}=a_{e}=\sqrt{D L^{3}}$, which is the effective aperture, then (6.16) is the same as (4.16).

\subsection{Imaging a passive point scatterer}

The generalization of the result (6.16) to the case of a passive point scatterer located at $\mathbf{y}=(0,0, L)$ is obtained in a similar way. We now use the expression of $\widehat{P}\left(\mathbf{x}_{p}, \mathbf{x}_{r}, \omega\right)$ for a passive point target with scattering coefficient $\tau(\omega)$ in (6.11) and we get

$$
\begin{gathered}
\Gamma_{P}^{I M}\left(\mathcal{O}_{r}, \mathcal{O}_{s}, \mathbf{y}^{s}\right)=\left(\frac{2}{h}\right)^{4} \int_{-a_{s} / 2}^{a_{s} / 2} \int_{-a_{s} / 2}^{a_{s} / 2} \int_{-a_{r} / 2}^{a_{r} / 2} \int_{-a_{r} / 2}^{a_{r} / 2} d x_{p} d x_{p}^{\prime} d x_{r} d x_{r}^{\prime} \\
\frac{1}{2 \pi} \int_{-\infty}^{\infty} d \omega|\widehat{f}(\omega)|^{2}|\widehat{\tau}(\omega)|^{2} \widehat{G}\left(\mathbf{x}_{r}, \mathbf{y}, \omega\right) \widehat{\widehat{G}\left(\mathbf{x}_{r}^{\prime}, \mathbf{y}, \omega\right)} \widehat{G}\left(\mathbf{x}_{p}, \mathbf{y}, \omega\right) \widehat{\widehat{G}\left(\mathbf{x}_{p}^{\prime}, \mathbf{y}, \omega\right)} e^{-i \omega\left(t_{p r}\left(\mathbf{y}^{s}\right)-t_{p^{\prime} r^{\prime}}\left(\mathbf{y}^{s}\right)\right)} .
\end{gathered}
$$

This is just like (6.12) but without integration over $\mathbf{y}, \mathbf{y}^{\prime}$ and we note that it involves the product of four (random) Green's functions.

To show that the imaging functional $\Gamma_{P}^{I M}\left(\mathcal{O}_{r}, \mathcal{O}_{s}, \mathbf{y}^{s}\right)$ is self-averaging and that only second moments of the Green's function are needed, let us consider

$$
\mathcal{A}_{p p^{\prime} r r^{\prime}}(t)=\int_{-\infty}^{\infty}|\widehat{f}(\omega)|^{2}|\widehat{\tau}(\omega)|^{2} \widehat{G}\left(\mathbf{x}_{p}, \mathbf{y}, \omega\right) \overline{\widehat{G}\left(\mathbf{x}_{p}^{\prime}, \mathbf{y}, \omega\right)} \widehat{G}\left(\mathbf{x}_{r}, \mathbf{y}, \omega\right) \overline{\widehat{G}\left(\mathbf{x}_{r}^{\prime}, \mathbf{y}, \omega\right)} e^{-i \omega t} d \omega,
$$

which appears in (6.17) evaluated at the deterministic time

$$
t=t_{p r}\left(\mathbf{y}^{s}\right)-t_{p^{\prime} r^{\prime}}\left(\mathbf{y}^{s}\right)
$$

We can write (6.18) as,

$$
\mathcal{A}_{p p^{\prime} r r^{\prime}}(t)=\int_{0}^{t} \Psi_{p p^{\prime}}(s) \Psi_{r r^{\prime}}(s-t) d s
$$

with

$$
\Psi_{p p^{\prime}}(t)=\int_{-\infty}^{\infty} \widehat{f}(\omega) \widehat{\tau}(\omega) \widehat{G}\left(\mathbf{x}_{p}, \mathbf{y}, \omega\right) \overline{\widehat{G}\left(\mathbf{x}_{p}^{\prime}, \mathbf{y}, \omega\right)} e^{-i \omega t} d \omega
$$

The process $\Psi_{p p^{\prime}}(t)$ is a weighted cross-correlation and is self-averaging because the large random phases of the Green's functions nearly cancel in (6.20). Since (6.19) is the convolution of $\Psi_{p p^{\prime}}(t)$ and $\Psi_{r r^{\prime}}(t)$, which are self averaging, we can replace $\Psi_{p p^{\prime}}(t)$ and $\Psi_{r r^{\prime}}(t-s)$ in (6.19) by their expectations and conclude that $\mathcal{A}_{p p^{\prime} r r^{\prime}}(t)$ is self-averaging with only second moments needed.

From (6.17) we obtain for a search point located at $\mathbf{y}^{s}=(\xi, 0, L+\eta)$,

$$
\Gamma^{I M}\left(\mathcal{O}_{r}, \mathcal{O}_{s}, \mathbf{y}^{s}\right) \approx \tilde{C}\left(L_{s}, a_{s}, L_{r}, a_{r}, \alpha\right) e^{-\frac{\xi^{2}}{2\left(L_{s}+\eta\right)^{2}}\left(\frac{L_{s}}{a_{e}^{s}}\right)^{2}} e^{-\frac{[\xi \cos \alpha-\eta \sin \alpha]^{2}}{2\left(L_{r}+[\xi \sin \alpha+\eta \cos \alpha]\right)^{2}}\left(\frac{L_{r}}{a_{e}^{r}}\right)^{2}},
$$

where $a_{e}^{s}=\sqrt{D L_{s}^{3}}$ and $a_{e}^{r}=\sqrt{D L_{r}^{3}}$ are the effective apertures associated with the source and receiver arrays, $L_{s}=L, L_{r}=L / \cos \alpha$. Note that (6.21) is the intersection of two cones with each 
one similar to the cone obtained for the active source case (see Figure 5 top) but with different axes of symmetry. Thus (6.21) indicates that using a source and a receiver array with offset angle $\alpha$ may allow the recovery of some range information for the target. It is not possible to obtain any range information, however, when the two arrays coincide, as we can see by taking $\alpha=0$ in (6.21),

$$
\Gamma_{P}^{I M}\left(\mathbf{y}^{s}\right) \approx \tilde{C}^{\prime \prime}(L, a) e^{-\frac{\xi^{2}}{(L+\eta)^{2}}\left(\frac{L}{a_{e}}\right)^{2}} .
$$

This expression is the square of (6.16) and this is because the random medium is traversed twice, from the array to the scatterer and from the scatterer back to the array. In other words, in the zero offset case $\alpha=0$ we sum twice over the same array. This is not necessary, however. In fact, when the receiver and transmitter arrays coincide, we can use only a column of the response matrix to image a passive point target. Taking $x_{p}=x_{0}$ fixed, a source at the central array element, we get

$$
\begin{aligned}
& \Gamma_{P}^{I M}\left(\mathbf{y}^{s}\right)=\frac{1}{2 \pi}\left(\frac{2}{h}\right)^{2} \int_{-a / 2}^{a / 2} \int_{-a / 2}^{a / 2} d x_{r} d x_{r}^{\prime} \int_{-\infty}^{\infty} d \omega|\widehat{f}(\omega)|^{2}|\widehat{\tau}(\omega)|^{2} \\
& \widehat{G}\left(\mathbf{x}_{r}, \mathbf{y}, \omega\right) \overline{\widehat{G}\left(\mathbf{x}_{r}^{\prime}, \mathbf{y}, \omega\right)} \widehat{G}\left(\mathbf{x}_{0}, \mathbf{y}, \omega\right) \overline{\widehat{G}\left(\mathbf{x}_{0}, \mathbf{y}, \omega\right)} e^{-i \omega\left(t_{0 r}\left(\mathbf{y}^{s}\right)-t_{0 r^{\prime}}\left(\mathbf{y}^{s}\right)\right)}
\end{aligned}
$$

which gives the same result (6.16) for an active source. This is because the large random phases of

the Green's functions $\widehat{G}\left(\mathbf{x}_{0}, \mathbf{y}, \omega\right) \widehat{G}\left(\mathbf{x}_{0}, \mathbf{y}, \omega\right)$ cancel exactly in (6.23), so that in (6.23) the blurring comes from traversing the random medium once, from the target to the array. This is the same as for an active source.

\subsection{Imaging the reflectivity of an extended scatterer}

We can use the same techniques to imaging the distributed reflectivity $\varrho(\mathbf{y})$. After some calculations similar to the ones above, given in [11], we get from (6.11)

$$
\begin{aligned}
\Gamma^{I M}\left(\mathcal{O}_{r}, \mathcal{O}_{s}, \mathbf{y}^{s}\right) & \approx \tilde{C}\left(L_{s}, a_{s}, L_{r}, a_{r}, \alpha\right) \\
& \int_{\mathcal{D}+\mathbf{y}_{0}} d y_{x} d y_{z} \varrho^{2}\left(y_{x}, y_{z}\right) e^{-\frac{\left(y_{x}-y_{x}^{s}\right)^{2}}{2\left(L_{s}+y_{z}^{s}\right)^{2}}\left(\frac{L_{s}}{a_{e}^{s}}\right)^{2}} e^{-\frac{\left[\left(y_{x}-y_{x}^{s}\right) \cos \alpha-\left(y_{z}-y_{z}^{s}\right) \sin \alpha\right]^{2}}{2\left(L_{r}+\left[y_{x}^{s} \sin \alpha+y_{z}^{s} \cos \alpha\right]\right)^{2}}\left(\frac{L_{r}}{a_{e}^{r}}\right)^{2}}
\end{aligned}
$$

for a search point located at $\mathbf{y}^{s}=\mathbf{y}_{0}+\left(y_{x}^{s}, 0, y_{z}^{s}\right)$ and for $\mathbf{y} \in \mathcal{D}$ parameterized by $\mathbf{y}=\mathbf{y}_{0}+\left(y_{x}, 0, y_{z}\right)$. The geometry of the problem is described in Figure 6 with the array element locations defined by (6.6) and where $a_{e}^{s}=\sqrt{D L_{s}^{3}}, L_{s}=L, a_{e}^{r}=\sqrt{D L_{r}^{3}}, L_{r}=L / \cos \alpha$.

This is the generalization of (6.21) to the case of an extended object with reflectivity $\varrho(\mathbf{y})$. We can analyze roughly the imaging resolution provided by the functional (6.24) when the overall range $L$ is assumed known and in a remote sensing regime, i.e.,

$$
\frac{\left|\mathbf{y}-\mathbf{y}_{0}\right|}{L} \ll 1, \forall \mathbf{y} \in \mathcal{D} \text {. }
$$

We also consider this functional in a domain of interest $\mathcal{D}_{I}$ probed by the search point $\mathbf{y}^{s}$ and which can be parameterized in the same way, that is, we also assume that

$$
\frac{\left|\mathbf{y}^{s}-\mathbf{y}_{0}\right|}{L} \ll 1, \forall \mathbf{y}^{s} \in \mathcal{D}_{I}
$$

Remark, that this assumption is not restrictive as it follows from (6.24) that only $\mathbf{y}^{s}$ satisfying

$$
\left|\mathbf{y}^{s}-\mathbf{y}_{0}\right| \leq a_{e}
$$


will contribute to a significant value of the functional $\Gamma^{I M}\left(\mathcal{O}_{r}, \mathcal{O}_{s}, \mathbf{y}^{s}\right)$.

In this regime we can approximate the denominators in the exponent of (6.24),

$$
\begin{aligned}
& L+y_{z}^{s} \approx L, \\
& L_{r}+\left[y_{x}^{s} \sin \alpha+y_{z}^{s} \cos \alpha\right] \approx L_{r} .
\end{aligned}
$$

and obtain

$$
\begin{aligned}
& \Gamma^{I M}\left(\mathcal{O}_{r}, \mathcal{O}_{s}, \mathbf{y}^{s}\right) \approx \tilde{C}\left(L_{s}, a_{s}, L_{r}, a_{r}, \alpha\right) \\
& \int_{\mathcal{D}+\mathbf{y}_{0}} d y_{x} d y_{z} \varrho^{2}\left(y_{x}, y_{z}\right) e^{-\frac{\left(y_{x}-y_{x}^{s}\right)^{2}}{2\left(a_{e}^{s}\right)^{2}}} e^{-\frac{\left[\left(y_{x}-y_{x}^{s}\right) \cos \alpha-\left(y_{z}-y_{z}^{s}\right) \sin \alpha\right]^{2}}{2\left(a_{e}^{r}\right)^{2}}} .
\end{aligned}
$$

Note, that we do not recover the reflectivity $\varrho(\mathbf{y})$ pointwise but rather $\varrho^{2}(\mathbf{y})$ averaged over an area defined by the intersection of two Gaussians centered at $\mathbf{y}^{s}$ and with variance proportional to the effective apertures. When two arrays are used then some range information remains in the matched field imaging functional, as it can be seen from (6.25). This is similar to the result obtained for the point scatterer case and suggests that it is necessary to have both an array of sources and an array of receivers in order to get some range resolution. When only one array is available, all range information is lost as we can easily verify from (6.25) by letting the offset angle be zero $(\alpha=0)$,

$$
\Gamma^{I M}\left(\mathbf{y}^{s}\right) \approx \tilde{C}(L, a) \int_{\mathcal{D}+\mathbf{y}_{0}} d y_{x} d y_{z} \varrho^{2}\left(y_{x}, y_{z}\right) e^{-\frac{\left(y_{x}-y_{x}^{s}\right)^{2}}{a_{e}^{2}}} .
$$

From the expression (6.25) we can also see that as the angle $\alpha$ decreases the variance of the Gaussian in the range direction increases. This expression (6.25) suggests also that for a symmetric geometry the reflectivity imaging functional satisfies a diffusion equation. We consider in the next section such a symmetric geometry setup.

\subsection{Symmetric geometry setup}

Consider a symmetric source-receiver setup with the two arrays of equal size $\left(a_{r}=a_{s}=a\right)$ as shown in Figure 7 . This can be viewed as a change in the coordinate system were the new origin is the center between the two arrays

$$
\mathbf{X}_{0}=\frac{\mathbf{O}_{s}+\mathbf{O}_{r}}{2}
$$

and in this case the array elements location is

$$
\begin{array}{ll}
\text { transmitter: } & \mathbf{x}_{p}=\left(\mathcal{O}_{s}+x_{p}, 0,0\right), x_{p}=p \frac{h}{2}, p=-N, \ldots, N, \\
\text { receiver: } & \mathbf{x}_{r}=\left(\mathcal{O}_{r}+x_{r}, 0,0\right), x_{r}=r \frac{h}{2}, r=-N_{r}, \ldots, N_{r} .
\end{array}
$$




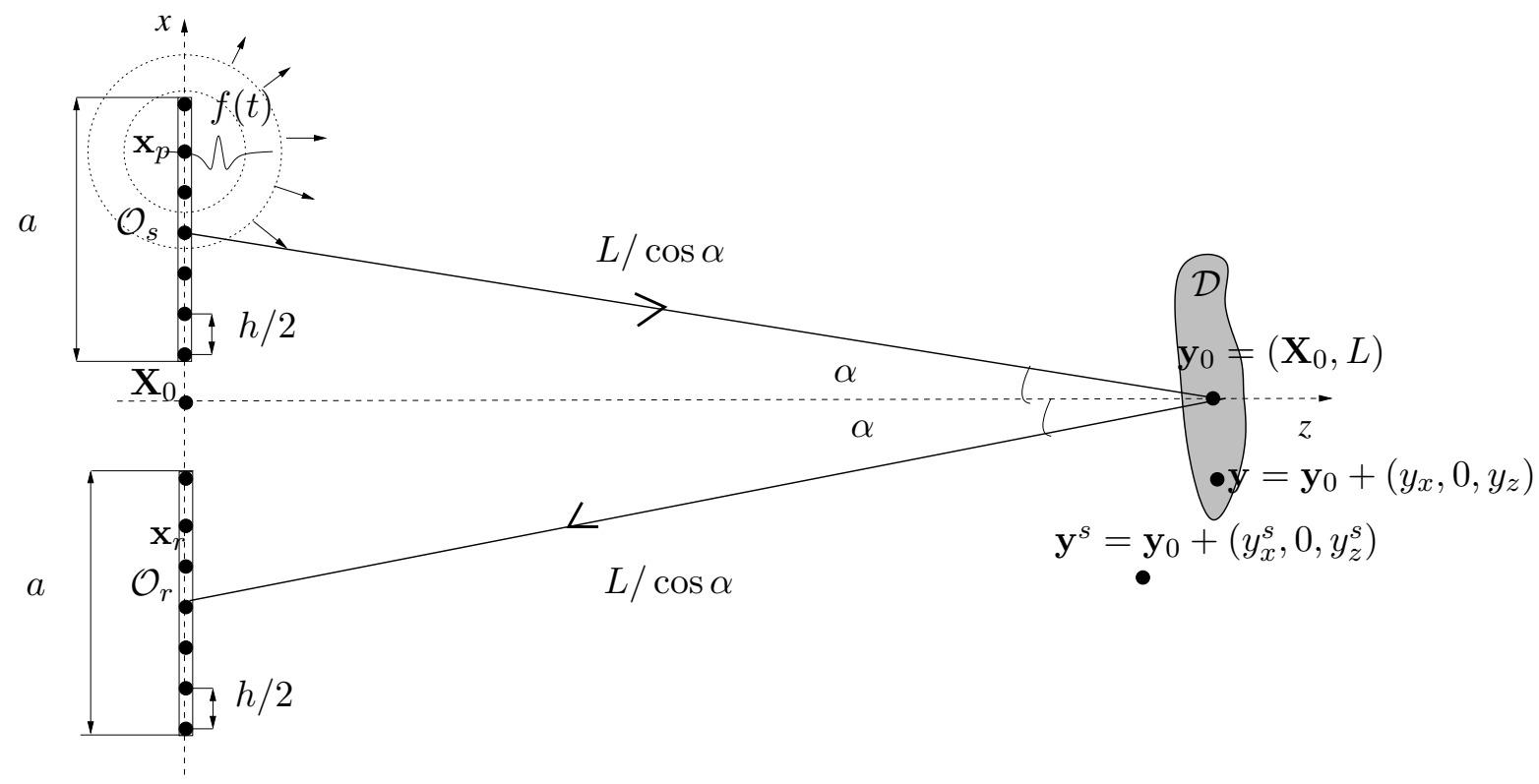

Figure 7: Symmetric geometry setup for distributed reflectivity imaging.

A reference point of $\mathcal{D}$ is located at

$$
\mathbf{y}_{0}=(L, 0,0)
$$

with respect to $\mathbf{X}_{0}$ and the distance from the central array points to $\mathbf{y}_{0}$ is $L / \cos \alpha$. For $\mathbf{y}^{s}=$ $\mathbf{y}_{0}+\left(y_{x}^{s}, 0, y_{z}^{s}\right)$ and $\mathbf{y}=\mathbf{y}_{0}+\left(y_{x}, 0, y_{z}\right),(6.25)$ becomes,

$$
\Gamma^{I M}\left(\mathbf{X}_{0}, \alpha, \mathbf{y}^{s}\right) \approx \tilde{C}(L, a, \alpha) \int_{\mathcal{D}+\mathbf{y}_{0}} d y_{x} d y_{z} \varrho^{2}\left(y_{x}, y_{z}\right) e^{-\frac{\left(y_{x}-y_{x}^{s}\right)^{2} \cos ^{2} \alpha+\left(y_{z}-y_{z}^{s}\right)^{2} \sin ^{2} \alpha}{D L^{3}} \cos ^{3} \alpha}
$$

If we let,

$$
I\left(\mathbf{y}^{s}, D, L, \alpha, \mathbf{X}_{0}\right)=\frac{1}{C(L, a, \alpha)} \Gamma^{I M}\left(L, \alpha, a, a_{e}, \mathbf{y}^{s}\right),
$$

then $I\left(\mathbf{y}^{s}, D, L, \alpha, \mathbf{X}_{0}\right)$ is the solution of a diffusion equation with anisotropic and singular coefficients,

$$
\begin{aligned}
& \frac{\partial I}{\partial D}=\frac{L^{3}}{\cos ^{3} \alpha}\left(\frac{1}{4 \cos ^{2} \alpha} \frac{\partial^{2} I}{\partial\left(y_{x}^{s}\right)^{2}}+\frac{1}{4 \sin ^{2} \alpha} \frac{\partial^{2} I}{\partial\left(y_{z}^{s}\right)^{2}}\right) \\
& \left.I\right|_{D=0}=\varrho^{2}\left(\mathbf{y}^{s}\right) .
\end{aligned}
$$

When a large aperture is available, the matched field functional $\Gamma^{I M}\left(\mathbf{X}_{0}, \alpha, \mathbf{y}^{s}\right)$ can be viewed as the result of pre-processing (coarse graining of the data). Specifically, by dividing the large array into smaller ones and by calculating local data covariances, we obtain $\Gamma^{I M}\left(\mathbf{X}_{0}, \alpha, \mathbf{y}^{s}\right)$ which is a blurry but stable view (image) of the reflectivity and can be described by (6.27) when the physical array aperture $a$ is large compared to the spot size $\sigma$, defined in (4.12) (cf. approximation (4.13)). The imaging resolution for the matched field method in random media is determined by the narrow-band effective aperture $a_{e}$, which does not depend on the physical aperture. Moreover, as (6.28) suggests, the optimal resolution in cross-range can be obtained for $\alpha=0$, and in range for $\alpha \approx 40^{\circ}$. Large synthetic apertures can be used to improve the imaging resolution of the matched 
field method by providing multiple blurry views of the reflectivity obtained for different sourcereceiver offset angles $\alpha$ and mid-points $\mathbf{X}_{0}$. One way to do this is to estimate $\varrho^{2}$ by a least squares deblurring method using data for several source-receiver offset angles $\alpha$ and mid-points $\mathbf{X}_{0}$.

We do not attempt to address the full inverse problem of imaging the reflectivity of an extended target in random media. Here, we focused attention on the matched field imaging functionals and we showed the importance of a key parameter, the effective aperture. Imaging of extended targets in random media is considered further in [11].

\section{Application to secure communication schemes}

The use of time reversal in wireless communication was suggested early in time-reversal experiments in the framework of underwater acoustics [35], [31], [26]. More recently, in [24, 22] experimental results using a Multiple Input Single Output (MISO) communication scheme with time reversal have been presented. We describe here this scheme. It is another important application of time reversal, as discussed in section 3.2, but now in communications. The estimation of $a_{e}$ in this framework allows us to assess at the transmitter the region around the receiver in which spatially localized, no interference communications through a cluttered medium can be realized.

A schematic view of the communication scheme is presented in Figure 8. The intended receiver

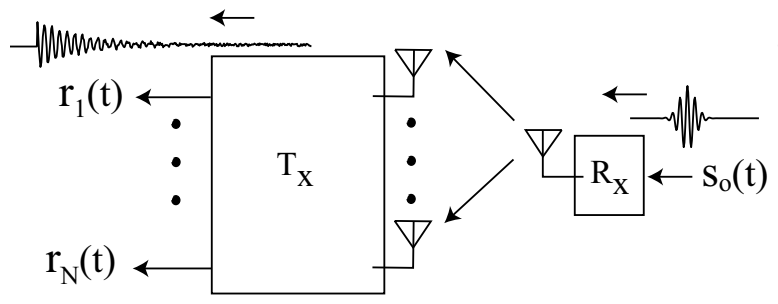

(a) The intended receiver first sends a pilot signal $s_{0}(t)$ to the transmitting array.

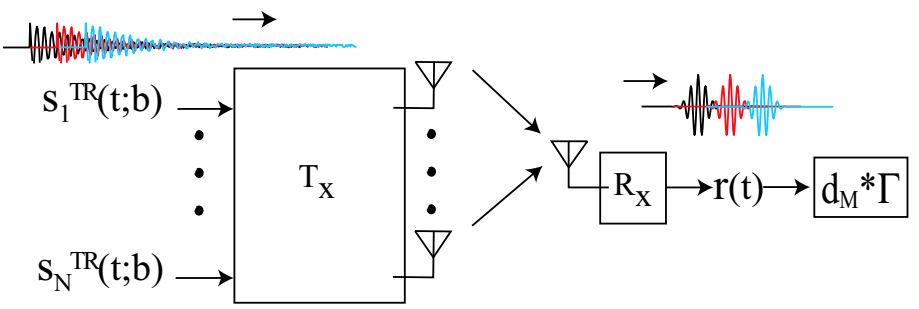

(b) Each element of the transmitting array records, timereverses and encodes a bitstream on the pilot signals received, and then sends them back.

Figure 8: The Multiple input Single Output time-reversal scheme.

first sends a pilot signal $s_{0}(t)=e^{-i \omega_{0} t} g(t)$ which is recorded by each element of the transmitter array. The modulating pulse is often the sinc function whose Fourier transform is

$$
\widehat{g}(\omega)=1, \text { for }|\omega| \leq 4 \pi W
$$

and zero outside, with $2 W$ the bandwidth. If we denote by $h_{p}(\mathbf{y}, t)$ the impulse response function between the receiver located at $\mathbf{y}$ and the $p$ - th element of the transmitter array then the signal recorded on the array is

$$
r_{p}(\mathbf{y}, t)=h_{p}(\mathbf{y}, t) \star_{t} s_{0}(t)+n_{p}(t) .
$$

Here $\star_{t}$ denotes convolution in time and $n_{p}(t)$ is the instrument white noise at the $p$ th element of the transmitting array. In the frequency domain (7.29) is

$$
\widehat{r}_{p}(\mathbf{y}, \omega)=\widehat{h}_{p}(\mathbf{y}, \omega) \widehat{s}_{0}(\omega)+\widehat{n}_{p}(\omega) .
$$

In the second step of this communication scheme the transmitter time-reverses the recorded signal, encodes a message on it and sends it back into the channel. The encoding scheme that we consider 
here is the BPSK (binary phase shift keying). Given a modulation pulse $s_{0}(t)$ a BPSK encoding scheme of an M-symbol data stream $\left\{b_{m}\right\}$ is

$$
d_{M}(t)=\sum_{m=1}^{M} b_{m} s_{0}(t-m \delta T)
$$

where $\delta T$ is the pulse repetition interval. The information is in the bitstream $\left\{b_{m}\right\}$, which are binary variables taking the values $(-1,1)$. In the frequency domain this scheme gives the signal

$$
\widehat{d}_{M}(\omega)=\widehat{s}_{0}(\omega) \sum_{m=1}^{M} b_{m} e^{-i m \omega \delta T},
$$

where $\widehat{d}_{M}(\omega)$ and $\widehat{s}_{0}(\omega)$ are the Fourier transforms of the message to be transmitted and the pilot pulse, respectively. Thus, for an M-symbol data stream the signal emitted from the $p-t h$ element of the transmitter is

$$
\widehat{s}_{p}(\mathbf{y}, \omega)=\overline{\widehat{r}_{p}(\mathbf{y}, \omega)} \sum_{m=1}^{M} b_{m} e^{-i m \omega \delta T}=\left(\overline{\widehat{h}_{p}(\mathbf{y}, \omega)} \overline{\widehat{s}_{0}(\omega)}+\overline{\widehat{n}_{p}(\omega)}\right) \sum_{m=1}^{M} b_{m} e^{-i m \omega \delta T},
$$

where the bar in (7.33) stands for complex conjugation. After propagation through the channel, the signal at a receiver located at $\mathbf{y}^{s}$ is,

$$
\begin{aligned}
\widehat{r}\left(\mathbf{y}^{s}, \mathbf{y}, \omega\right) & =\sum_{p=1}^{N_{T}} \widehat{h}_{p}\left(\mathbf{y}^{s}, \omega\right) \widehat{s}_{p}(\mathbf{y}, \omega)+\widehat{n}_{0}(\omega) \\
= & \left(\sum_{p=1}^{N_{T}} \widehat{h}_{p}\left(\mathbf{y}^{s}, \omega\right) \overline{\widehat{h}_{p}(\mathbf{y}, \omega)} \overline{\widehat{s}_{0}(\omega)}+\sum_{p=1}^{N_{T}} \widehat{h}_{p}\left(\mathbf{y}^{s}, \omega\right) \overline{\widehat{n}_{p}(\omega)}\right) \sum_{m=1}^{M} b_{m} e^{-i m \omega \delta T}+\widehat{n}_{0}(\omega) .
\end{aligned}
$$

where $\widehat{n}_{0}(\omega)$ is an additional noise term at receiver. Allowing the receiver to move from $\mathbf{y}$ to $\mathbf{y}^{s}$ probes the spatial focusing properties of the communication schemes.

To interpret the message the receiver correlates $r\left(\mathbf{y}^{s}, \mathbf{y}, t\right)$ with the pilot pulse $s_{0}(t)$. In the frequency domain, the resulting signal is

$$
\widehat{S}\left(\mathbf{y}^{s}, \mathbf{y}, \omega\right)=\left(\widehat{\Gamma}\left(\mathbf{y}^{s}, \mathbf{y}, \omega\right)+\sum_{p=1}^{N_{T}} \widehat{h}_{p}\left(\mathbf{y}^{s}, \omega\right) \overline{\widehat{n}_{p}(\omega)}\right) \widehat{d}_{M}(\omega)+\widehat{s}_{0}(\omega) \widehat{n}_{0}(\omega),
$$

where $\widehat{\Gamma}\left(\mathbf{y}^{s}, \mathbf{y}, \omega\right)$ is the response function of the communication scheme,

$$
\widehat{\Gamma}\left(\mathbf{y}^{s}, \mathbf{y}, \omega\right)=\sum_{p=1}^{N_{T}} \widehat{h}_{p}\left(\mathbf{y}^{s}, \omega\right) \overline{\widehat{h}_{p}(\mathbf{y}, \omega)} \overline{\widehat{s}_{0}(\omega)}
$$

and $\widehat{d}_{M}(\omega)$ is the M-symbol data stream carried by the pilot pulse, defined by (7.32). In an infinite random medium the transfer function $\widehat{h}_{p}(\mathbf{y}, \omega)$ is the two point Green's function $\widehat{G}\left(\mathbf{x}_{p}, \mathbf{y}, \omega\right)$ satisfying the reduced wave equation (2.4) and we have

$$
\widehat{\Gamma}\left(\mathbf{y}^{s}, \mathbf{y}, \omega\right)=\widehat{\Gamma}^{T R}\left(\mathbf{y}^{s}, \omega\right)=\widehat{\Gamma}_{0}^{T R}\left(\mathbf{y}^{s}, \omega\right) e^{-\frac{k^{2} a_{e}^{2} \xi^{2}}{2 L^{2}}},
$$

for $\mathbf{y}^{s}=\mathbf{y}+(\xi, 0, L), s_{0}(t)=f(t)$. When the signal to noise power is high, that is, when the noise terms $\widehat{n}_{p}$ and $\widehat{n}_{0}$ can be neglected, then we can apply the theoretical results of section 3.2. 
In particular we can use the method of section 5.2 to estimate the effective aperture $a_{e}$ at the transmitter and predict the refocusing spot size from (3.17). This provides a robust estimate at the transmitter of the area in which the intended receiver can move without losing reception of the message, and/or the area around the receiver in which the message can be intercepted.

Another reason for using time-reversal in communications, in addition to spatial localization at the receiver, is the following. Time reversal is a temporal matched filter so the indented receiver can be very simple and need not use sophisticated decoding. If we let $\xi=0$ in (7.37) we see that the signal at the receiver (for $\mathbf{y}=\mathbf{y}^{s}$ ) has no random phase, even when transmission is through a cluttered (random) medium. This means that inter-symbol interference is reduced significantly because of the time-reversal process.

This simple MISO time-reversal coding scheme achieves signal compression both in time and space allowing for secure communications with reduced inter-symbol interference. Moreover, the rate of information transmission for this scheme realizes the Shannon capacity for a flat frequency channel that is known at the transmitter [40]. For a frequency selective MISO channel, as we have in random media, the Shannon capacity is not realized unless a more elaborate frequency dependent coding scheme [40] is used. We compare the performance of time reversal communication schemes with other MISO (multiple input single output) and SIMO (single input multiple output) communications schemes in rich scattering environments (clutter) in [9]. Numerical results illustrating the efficiency of time-reversal and generalized time reversal coding schemes when the receiver has perfect channel knowledge (and the signal to noise ration is high) are presented in [34].

Perfect channel knowledge means that the transfer function $\widehat{h}_{p}(\mathbf{y}, \omega)$ remains the same between the first and the second step of the communication scheme, which may not be true if, for example, the propagation medium changes in time or if the transmitter knows the transfer function up to an error [40]. We are currently working on this problem as well as on the generalization of the time-reversal process to MIMO (multiple input multiple output) communication schemes. As for the MISO scheme, depending on the parameters of the problem, we expect the narrow-band or broad-band effective aperture to play an important role for communications trough clutter.

\section{Summary}

We have analyzed the matched field imaging functional, which can be used for locating small active scatterers embedded in a random medium. In a remote sensing regime with significant multipathing we obtained an analytical expression for the imaging functional. Using this expression we have quantified the imaging resolution in random media and we have shown that this depends on a single parameter, the narrow-band effective aperture $a_{e}$. Using this for the matched field functional we have proposed a simple and robust method for estimating the unknown effective aperture $a_{e}$. We have assessed the feasibility of our estimation approach with direct numerical simulations. In particular, using the estimated $a_{e}$ we can predict the refocused spot size for time reversal in random media. We have seen remarkably good agreement between the predicted and the observed refocused spot size in direct numerical simulations of time reversal in a random medium. We have also indicated how the concept of effective aperture enters in matched field or interferometric functionals for the reflectivity of an extended scatterer as well as in wireless communications with time reversal. 


\section{A The white noise limit and the parabolic approximation}

We collect here some comments on the scaling analysis of the random Helmholtz equation (2.4) and refer to $[1,36,44]$ for additional comments and results on scaling and asymptotics in the high-frequency and white-noise regime.

Let $L_{z}$ be a range scale and $L_{x}$ a cross-range scale and defile the dimensionless parameters $\epsilon=l / L_{z}, \delta=l / L_{x}$, where $l$ is the correlation length of the fluctuations in the index of refraction. Let $\gamma=1 / k_{0} l$. We also define the Fresnel number

$$
\theta=\frac{L_{z}}{k_{0} L_{x}^{2}}=\gamma \frac{\delta^{2}}{\epsilon}
$$

Then the scaled Helmholtz equation, with the phase $e^{i k z}$ removed, is

$$
\frac{\epsilon^{2} \theta^{2}}{\delta^{2}} \psi_{z z}+2 i k \theta \psi_{z}+\theta^{2} \Delta_{x} \psi+\frac{k^{2} \delta}{\epsilon^{1 / 2}} \mu\left(\frac{\mathbf{x}}{\delta}, \frac{z}{\epsilon}\right) \psi=0 .
$$

Here we relate the strength of the fluctuations $\sigma$ to $\epsilon$ and $\delta$ by $\epsilon=\sigma^{2 / 3} \delta^{2 / 3}$. It is in this regime that fluctuation effects have a fully developed form.

We first need to examine when the parabolic approximation is valid. It is in the white noise limit $\epsilon \rightarrow 0$, with Fresnel number $\theta$ and $\delta$ fixed, that the parabolic approximation is valid as was pointed out in [1]. This is easily seen if the random fluctuations $\mu$ are differentiable in $z$. The parabolic approximation is clearly not valid in the high frequency limit $\theta \rightarrow 0$, before the white noise limit $\epsilon \rightarrow 0$ is also taken. In the white noise limit, the wave function $\psi(z, \mathbf{x})$ satisfies an Ito-Schrödinger equation

$$
2 i k \theta d_{z} \psi+\theta^{2} \Delta_{x} \psi d z+\frac{i k^{3} \delta^{2}}{4 \theta} R_{0}(0) \psi d z+k^{2} \delta \psi d_{z} B\left(\frac{\mathbf{x}}{\delta}, z\right)=0 .
$$

Here $R_{0}$ is the integrated covariance of the fluctuations $\mu$ and the Brownian field $B(\mathbf{x}, z)$ has covariance

$$
\left\langle B\left(\mathbf{x}, z_{1}\right) B\left(\mathbf{y}, z_{2}\right)\right\rangle=R_{0}(\mathbf{x}-\mathbf{y}) z_{1} \wedge z_{2} .
$$

This Ito-Schrödinger equation is the result of the central limit theorem applied to (A.1). Let

$$
B^{\epsilon}(\mathbf{x}, z)=\frac{1}{\sqrt{\epsilon}} \int_{0}^{z} \mu\left(\mathbf{x}, \frac{s}{\epsilon}\right) d s .
$$

Then, as $\epsilon \rightarrow 0$ this process converges weakly, under suitable hypotheses, to the Brownian field $B(\mathbf{x}, z)$ with the above covariance. The extra term in (A.2) is the Stratonovich correction. It is from this stochastic equation that the moment formula that we use in the paper is obtained.

The white noise limit for stochastic partial differential equations is analyzed in [15] and a rigorous theory of the Ito-Schrödinger equation is given in [20]. The ergodic theory of the ItoSchroedinger equation is explored in [33]. Wave propagation in the parabolic approximation with white-noise fluctuations is considered in detail in [29, 43].

\section{Acknowledgments}

The work of L. Borcea was partially supported by the Office of Naval Research, under grant N00014-02-1-0088 and by the National Science Foundation, grant DMS-0305056. The work of G. Papanicolaou was supported by grants AFOSR F49620-01-1-0465, ONR N00014-02-1-0088 and 02SC-ARO-1067-MOD 1. The work of C. Tsogka was partially supported by the Office of Naval Research, under grant N00014-02-1-0088. 


\section{References}

[1] F. Bailly, J.F. Clouet, and J.P. Fouque. Parabolic and white noise approximation for waves in random media. SIAM J.Appl. Math., 56:1445-1470, 1996.

[2] G. Bal, G. C. Papanicolaou, and L. Ryzhik. Self-averaging in time reversal for the parabolic wave equation. Stochastics and Dynamics, 2:507-531, 2002.

[3] E. Bécache, P. Joly, and C. Tsogka. Etude d'un nouvel élément fini mixte permettant la condensation de masse. C. R. Acad. Sci. Paris Sér. I Math., 324:1281-1286, 1997.

[4] E. Bécache, P. Joly, and C. Tsogka. An analysis of new mixed finite elements for the approximation of wave propagation problems. SIAM J. Numer. Anal., 37:1053-1084, 2000.

[5] J.P. Bérenger. A perfectly matched layer for the absorption of electromagnetic waves. $J$. Comput. Phys., 114:185-200, 1994.

[6] J. Berryman, L. Borcea, G. C. Papanicolaou, and C. Tsogka. Statistically stable ultrasonic imaging in random media. Journal of Acoustical Society of America, 112:1509-1522, 2002.

[7] N. Bleistein, J.K. Cohen, and J.W. Stockwell Jr. Mathematics of multidimensional seismic imaging, migration, and inversion. Springer, New York, 2001.

[8] P. Blomgren, G. Papanicolaou, and H. Zhao. Super-resolution in time-reversal acoustics. Journal of the Acoustical Society of America, 111:238-248, 2002.

[9] L. Borcea, A. D. Kim, G. C. Papanicolaou, and C. Tsogka. Communications in cluttered media with active and passive phase conjugation. preprint, 2003.

[10] L. Borcea, G. Papanicolaou, and C. Tsogka. A resolution study for imaging and time reversal in random media. To appear in Contemporary Math, 2003.

[11] L. Borcea, G. C. Papanicolaou, and C. Tsogka. Imaging the reflectivity of extended scatterers in random media. preprint, 2003.

[12] L. Borcea, G. C. Papanicolaou, C. Tsogka, and J. Berryman. Imaging and time reversal in random media. Inverse Problems, 18:1247-1279, 2002.

[13] B. Borden. Mathematical problems in radar inverse scattering, topical review. Inverse Problems, 18:R1-R28, 2002.

[14] M. Born and E. Wolf. Principles of optics. Academic Press, New York, 1970.

[15] R. Bouc and E. Pardoux. Asymptotic analysis of pdes with wide-band noise disturbances and expansion of the moments. Stochastic Analysis and Applications, 2:369-422, 1984.

[16] L. Carin, N. Geng, M. McClure, Y. Dong, Z. Liu, J. He, J. Sichina, M. Ressler, L. Nguyen, and A. Sullivan. Wide-area detection of land mines and unexploded ordnance. Inverse Problems, 18:575-609, 2002.

[17] J. F. Claerbout. Fundamentals of geophysical data processing : with applications to petroleum prospecting. CA : Blackwell Scientific Publications, Palo Alto, 1985. 
[18] J.F. Clouet and J.P. Fouque. A time-reversal method for an acoustical pulse propagating in randomly layered media. Wave Motion, 25:361-368, 1997.

[19] J.C. Curlander and R.N. McDonough. Synthetic Aperture Radar. Wiley, New York, 1991.

[20] D. Dawson and G. Papanicolaou. A random wave process. Appl. Math. Optim., 12:97-114, 1984.

[21] A. Derode, P. Roux, and M. Fink. Robust acoustic time reversal with high-order multiple scattering. Phys. Rev. Let., 75(23):4206-4210, 1995.

[22] A. Derode, A. Tourin, J. de Rosny, M. Tanter, S. Yon, and M. Fink. Taking advantage of multiple scattering to communicate with time-reversal antennas. Phys. Rev. Letters, 90(014301), 2003.

[23] D. R. Dowling and D. R. Jackson. Narrow band performance of phase conjugate arrays in dynamic random media. J. Acoust. Soc. Am., 91:3257-3277, 1992.

[24] G. F. Edelman, T. Akal, W. S. Hodgkiss, S. Kim, W. A. Kuperman, and H. C. Song. An initial demontration of underwater acoustic communication using time reversal. IEEE J. of Oceanic Engr., 27:602-609, 2002.

[25] M. Fink. Time reversal mirrors. J. Phys. D, 26:1330-1350, 1993.

[26] M. Fink. Time reversed acoustics. Phys. Today, 50:34-40, 1997.

[27] M. Fink and C. Prada. Acoustic time-reversal mirrors, topical review. Inverse Problems, 17:R1-R38, 2001.

[28] J. P. Fouque and K. Solna. Time-reversal aperture enhancement. preprint, 2000.

[29] K. Furutsu. Random Media and Boundaries: Unified Theory, Two-Scale Method, and Applications. Springer Verlag, 1993.

[30] S. Haykin, J. Litva, and T. J. Shepherd. Radar Array Processing. Springer-Verlag, New York, 1993.

[31] W. S. Hodgkiss, H. C. Song, W. A. Kuperman, T. Akal, C. Ferla, and D. R. Jackson. A long range and variable focus phase-conjugation experiment in swallow water. J. Acoust. Soc. Am., 105:1597-1604, 1999.

[32] A. Ishimaru. Wave Propagation and Scattering in Random Media. IEEE Press and Oxford University Press, 1997.

[33] G.C. Papanicolaou J.P. Fouque and Y. Samuelides. Forward and markov approximation: The strong intensity fluctuations regime revisited. Waves in Random Media, 8:303-314, 1988.

[34] A. Kim, P. Blomgren, and G. C. Papanicolaou. Communications in a complex environment using time reversal. preprint, 2003.

[35] W. A. Kuperman, W. S. Hodgkiss, H. C. Song, T. Akal, C. Ferla, and D. R. Jackson. Phase conjugation in the ocean : Experimental demonstration of an acoustic time reversal mirror. $J$. Acoust. Soc. Am., 103:25-40, 1998. 
[36] B. Nair and B. White. High-frequency wave propagation in random media- a unified approach. SIAM J. Appl. Math., 51:374-411, 1991.

[37] C. J. Nolan and M. Cheney. Synthetic aperture inversion. Inverse Problems, 18(1):221-235, 2002 .

[38] G. Papanicolaou, L. Ryzhik, and K. Solna. Statistical stability in time reversal. To appear in the SIAM J. Applied Mathematics, 2003.

[39] G. C. Papanicolaou, L. Ryzhik, and K. Solna. The parabolic wave approximation and time reversal. Matematica Contemporanea, 23:139-160, 2002.

[40] A. Paulraj, R. Nabar, and D. Gore. Introduction to Space-Time Wireless Communications. Cambridge University Press, 2003.

[41] S.M. Rytov, Yu.A. Kravtsov, and V.I. Tatarskii. Principles of Statistical Radiphysics. Springer Verlag, 1989.

[42] H. C. Song, W. A. Kuperman, and W. S. Hodgkiss. Iterative time reversal in the ocean. J. Acoust. Soc. Am., 105:3176-3184, 1999.

[43] V. I. Tatarskii, A. Ishimaru, and V. U. Zavorotny, editors. Wave Propagation in Random Media (Scintillation). SPIE and IOP, 1993.

[44] B. White. The stochastic caustic. SIAM J. Appl. Math., 44:127-149, 1984. 